Pure and Applied Mathematics Quarterly

Volume 1, Number 4

(Special Issue: In Memory of

Armand Borel, Part 3 of 3 )

$755-789,2005$

\title{
Spherical Unitary Principal Series
}

\author{
Dan Barbasch and Dan Ciubotaru
}

\section{INTRODUCTION}

In this paper, we prove two results about the unitary dual of graded affine Iwahori-Hecke algebras. The first one, theorem 2.4, is in the case of the Hecke algebra with equal parameters which arises from split p-adic groups. It says that multiplicities of W-types in irreducible spherical modules are constant over the faces of root hyperplane arrangements. This result is the basis for an algorithm to compute the spherical dual of the graded affine Iwahori-Hecke algebra.

The second result is in the case of graded Iwahori-Hecke algebras with unequal parameters of type B/C. We determine all the irreducible principal series which are unitary.

These results have consequences for the determination of the spherical unitary dual of p-adic groups, as well as real groups. An example is in section 4 .

The starting point is Borel and Casselman's theorem establishing an equivalence of categories between the category of Iwahori-spherical representations and finite dimensional representations of the Iwahori-Hecke algebra.

1.1. Let $\mathbb{F}$ be a p-adic field. Let $G$ be the $\mathbb{F}$-rational points of a connected linear algebraic reductive group defined over $\mathbb{F}$. We assume that $G$ is split. Denote by $A$ a maximal split torus, and fix a Borel subgroup with Levi decomposition $B=A N$. Let

$$
\begin{aligned}
& \mathcal{R}=\{x \in \mathbb{F}:|x| \leq 1\}, \quad \mathcal{P}=\{x \in \mathbb{F}:|x|<1\}, \\
& \Omega=\{x \in \mathbb{F}:|x|=1\} .
\end{aligned}
$$


Then $G$ has a maximal compact subgroup $K=G(\mathcal{R})$, and there is an exact sequence

$$
1 \longrightarrow K_{1} \longrightarrow K \longrightarrow G(\mathcal{R} / \mathcal{P}) \cong G\left(\mathbb{F}_{q}\right) \longrightarrow 1
$$

A character $\nu \in \hat{A}$ is called unramified, if its restriction to $A \cap K$ is trivial. The principal series $I(\nu)$ is the Harish-Chandra induced module from the character $\nu$. It is normalized so that $I(\nu)$ is unitary whenever $\nu$ is unitary. Fix a Borel subgroup in $G\left(\mathbb{F}_{q}\right)$. Then its inverse image is an open compact subgroup called the Iwahori subgroup and denoted by $\mathcal{I}$. The space of compactly supported biinvariant functions $\mathcal{H}:=\mathcal{H}(\mathcal{I} \backslash G / \mathcal{I})$ is an algebra under convolution

$$
(f \star g)(x):=\int_{G} f\left(x y^{-1}\right) g(y) d y,
$$

and is called the Iwahori-Hecke algebra. It has a $*$ operation, as follows: for $f \in \mathcal{H}$,

$$
f^{*}(x):=\overline{f\left(x^{-1}\right)}
$$

If $(\pi, V)$ is an admissible module for $G$, consider

$$
V^{\mathcal{I}}=\{v \in V: \pi(x) v=v, \text { for all } x \in \mathcal{I}\} .
$$

$\mathcal{H}$ acts on $V^{\mathcal{I}}$ by the formula

$$
\pi(f) v:=\int_{G} f(x) \pi(x) v d x .
$$

Let

$\mathcal{C}(\mathcal{I})=$ the category of admissible finite length representations so that all their subquotients are generated by their Iwahori fixed vectors,

$\mathcal{C}(\mathcal{H})=$ the category of finite dimensional representations of $\mathcal{H}$.

The study of $I(\nu)$ and its composition factors is important for harmonic analysis and automorphic forms. The following theorem, due to A. Borel, is fundamental for the study of the unramified principal series, and its composition factors.

Theorem. ([B])

(1) The functor $V \mapsto V^{\mathcal{I}}$ is an equivalence of categories from $\mathcal{C}(\mathcal{I})$ to $\mathcal{C}(\mathcal{H})$. The inverse is given by $W \mapsto \mathcal{H}(G / \mathcal{I}) \otimes W$, where $\mathcal{H}(G / \mathcal{I})$ is the algebra (under convolution) of smooth compactly supported right $\mathcal{I}$-invariant functions.

(2) An irreducible representation $\pi$ is in $\mathcal{C}(\mathcal{I})$ if and only if it is a subquotient of an $I(\nu)$ with $\nu$ unramified. 
This result was also proved independently by Casselman, and had a profound influence on the representation theory of p-adic groups. The work of $[\mathrm{BK}]$ and $[\mathrm{HM}]$ uses this idea as the basis for their classification of irreducible admissible modules of $G L(n)$. The general strategy is to prove a far reaching generalization of theorem 1.1, namely that the category of admissible representations decomposes into blocks with the following properties. Each block is parametrized by a pair $(\mathcal{J}, \sigma)$, where $\sigma$ is an irreducible representation of a compact open subgroup $\mathcal{J}$. An irreducible representation $(\pi, V)$ belongs to the block parametrized by $(\mathcal{J}, \sigma)$ if and only if $\operatorname{Hom}_{\mathcal{J}}[\pi, \sigma] \neq 0$. One of the goals is then to show that the analogue of theorem 1.1 holds. The Iwahori-Hecke algebra $\mathcal{H}$ is replaced by $\mathcal{H}(\sigma \backslash G / \sigma)$, compactly supported vector valued functions which transform according to $\sigma$ on the left and right. Further work in this direction has been done by $[\mathrm{K}],[\mathrm{Y}]$, and others (see references therein).

Since $\mathcal{H}$ has a star operation, it makes sense to talk about Hermitian and unitary modules. It is more or less clear that an admissible representation $V \in$ $\mathcal{C}(\mathcal{I})$ of $G$ is Hermitian if and only if $V^{\mathcal{I}}$ is Hermitian. It is also clear that if $V$ is unitary, then so is $V^{\mathcal{I}}$. However the converse is not so trivial. It was conjectured some time ago by Borel and Casselman, as well as others. The subject of two papers [BM1] and [BM2] is to prove this conjecture. The result is summarized in the next result.

Proposition ([BM1], [BM2]). An irreducible representation in $\mathcal{C}(\mathcal{I})$ is unitary if and only if $V^{\mathcal{I}}$ is unitary.

The idea of the proof is to combine the results of [KL], [L2], [L4] and [L5], which give a precise classification of the irreducible modules in $\mathcal{C}(\mathcal{H})$, with techniques from the real groups, in [V1], on signatures of the Hermitian forms of irreducible modules.

This paper is organized as follows. In sections 2.1 and 2.2 we review the graded affine Hecke algebra, and the classification of irreducible modules due to Kazhdan and Lusztig. Sections 2.3 and 2.4 apply these results to the spherical case. Theorem 2.4 has been known to the authors for some time. It is basic for establishing an algorithm for determining the spherical unitary dual of any Hecke algebra with equal parameters. More details are in the Remark in section 2.12. We have implemented this algorithm using mathematica, and it played a role in our determination of the spherical unitary dual of all Iwahori-Hecke algebras of exceptional types. The results for type $F_{4}$ are in $[C]$, while for type $E$, they are in $[\mathrm{BC}]$. The algorithm was also implemented by J. Adams, J.-K. Yu, and J. Stembridge. More information can be found at atlas.math.umd.edu. 
Sections 2.6-2.12 recall results about the * operation for graded affine algebras, the Langlands classification and its relation to the Kazhdan-Lusztig classification. Sections 2.11 and 2.12 are particularly relevant for the spherical unitary dual.

Sections 3.1-3.3 introduce the graded affine Hecke algebras with unequal parameters. The main result is theorem 3.2, which says that any generic spherical module (beginning of section 3.2) is unitary if and only if it is part of a complementary series. The explicit set of parameters is given in theorem 3.6. The proof is given in sections 3.3-3.8. Theorems 3.2 and 3.6 generalize results in [Ba2], where a full description of the spherical unitary dual of the classical groups is given.

Theorem 3.6 gives necessary conditions for spherical representations to be unitary in the case of p-adic groups of type $B / C$. But also, as described in [Ba1], these results have consequences for the unitary dual of the real groups $U(p, q)$. Such an application is given in section 4 .

This material is based upon work supported by NSF grants DMS-9706758, DMS0070561 and DMS-03001712.

\section{Split Groups}

2.1. The Graded Hecke Algebra. The Hecke algebra $\mathcal{H}$ can be described by generators and relations. Denote by ${ }^{L} G$ the (complex) dual group of $G$, with maximal torus ${ }^{L} A$ and Borel subgroup ${ }^{L} B$ containing ${ }^{L} A$. We emphasize that the root datum of the Hecke algebra is the one for the dual complex group ${ }^{L} G$, and that the roots $\alpha$ will be the roots of ${ }^{L} G$. Let $z$ be an indeterminate (which can then be specialized to $q^{1 / 2}$ ). Let $\Pi \subset R^{+} \subset R$ be the set of simple coroots, positive coroots, respectively coroots corresponding to the split Cartan subgroup $A$ inside the Borel subgroup $B$ from section 1.1. Denote by $S$ the simple root reflections. $G_{m}=G L(1, \mathbb{F})$ and $\mathcal{X}=\check{\mathcal{Y}}=\operatorname{Hom}\left(G_{m}, A\right)$ be the (algebraic) lattice of 1-parameter subgroups and $\mathcal{Y}=\check{\mathcal{X}}=\operatorname{Hom}\left(A, G_{m}\right)$ the lattice of algebraic characters. Then $\mathcal{H}$ can be characterized as the Hecke algebra over $\mathbb{C}\left[z, z^{-1}\right]$ attached to the root datum $\mathcal{R}=(\mathcal{Y}, \mathcal{X}, \check{R}, R, \check{\Pi})$. The set of generators we will use is the one first introduced by Bernstein. Let

$$
\mathcal{A}=\text { rational functions on } \mathbb{C}^{*} \times{ }^{L} A \text {. }
$$


Then $\mathcal{H}$ is generated (over $\mathbb{C}\left[z, z^{-1}\right]$ ) by $\left\{T_{w}\right\}_{w \in W}$ and $\left\{\theta_{x}\right\}_{x \in \mathcal{X}}$, subject to the relations

$$
\begin{aligned}
T_{w} T_{w^{\prime}} & =T_{w w^{\prime}} \quad\left(l(w)+l\left(w^{\prime}\right)=l\left(w w^{\prime}\right)\right), \\
\theta_{x} \theta_{y} & =\theta_{x+y}, \\
T_{s}^{2} & =\left(z^{2}-1\right) T_{s}+z^{2} \\
\theta_{x} T_{s} & =T_{s} \theta_{s x}+\left(z^{2}-1\right) \frac{\theta_{x}-\theta_{s x}}{1-\theta_{\alpha}} .
\end{aligned}
$$

This realization is very convenient for determining the center of $\mathcal{H}$ and thus computing infinitesimal characters of representations.

Proposition (Bernstein-Lusztig). The center of $\mathcal{H}$ is given by the Weyl group invariants in $\mathcal{A}$.

In particular, infinitesimal characters are parametrized by $W$-orbits $\chi=(q, t) \in$ $\mathbb{C}^{*} \times{ }^{L} A$. We always assume that $q$ is real or at least not a root of unity. In particular, such an infinitesimal character is called real if $t$ is hyperbolic. The subject of [BM2] is to show that the classification of unitary irreducible modules of $\mathcal{H}$ with infinitesimal character $\chi$ corresponding to $(q, t)$ is equivalent to the classification of the unitary dual for real infinitesimal character. Thus we will assume from here on that the infinitesimal character is always real. The study of representations of $\mathcal{H}$ can be simplified by using the graded Hecke algebra introduced by Lusztig. Let

$$
\mathcal{J}=\{f \in \mathcal{A}: f(1,1)=0\}
$$

This is an ideal in $\mathcal{A}$ and it satisfies $\mathcal{H} \mathcal{J}=\mathcal{J H}$. Set $\mathcal{H}^{i}=\mathcal{H} \cdot \mathcal{J}^{i}$ (the ideal $\mathcal{J}^{i}$ consists of the functions which vanish to order at least $i$ at $(1,1)$ ). We can introduce the filtration

$$
\mathcal{H}=\mathcal{H}^{0} \supset \cdots \supset \mathcal{H}^{i} \supset \mathcal{H}^{i+1} \supset \ldots,
$$

and form the graded object $\mathbb{H}$. It can be written as

$$
\mathbb{H}=\mathbb{C}[\mathbf{r}] \otimes \mathbb{C}[W] \otimes \mathbb{A},
$$

where $\mathbf{r} \equiv z-1(\bmod \mathcal{J})$, and $\mathbb{A}$ is the symmetric algebra over $\mathfrak{a}=\mathcal{X} \otimes_{\mathbb{Z}} \mathbb{C}$. The previous relations become

$$
\begin{aligned}
t_{w} t_{w^{\prime}} & =t_{w w^{\prime}}, \\
t_{s}^{2} & =1, \\
t_{s} \omega & =s(\omega) t_{s}+2 \mathbf{r}\langle\omega, \check{\alpha}\rangle, \quad s=s_{\alpha}, \omega \in \mathfrak{a} .
\end{aligned}
$$

The center of $\mathbb{H}$ is $\mathbb{C}[\mathbf{r}] \otimes \mathbb{A}^{W}$. Let ${ }^{L} \mathfrak{a}=\mathcal{Y} \otimes_{\mathbb{Z}} \mathbb{C}$ be the Cartan subalgebra of ${ }^{L} G$. The infinitesimal characters are parametrized by $W$-orbits of elements $\bar{\chi}=$ $(r, t) \in \mathbb{C} \times{ }^{L} \mathfrak{a}$ (the indeterminate $\mathbf{r}$ for $\mathbb{H}$ acts by $r$ ). Then $\chi=\left(e^{r}, e^{t}\right) \in \mathbb{C}^{*} \times{ }^{L} A$ is an infinitesimal character for $\mathcal{H}$. Let $\mathcal{I}_{\chi}$ be the kernel of $\chi$ on the center of $\mathcal{H}$. Then $\mathcal{H} \cdot \mathcal{I}_{\chi}$ is an ideal in $\mathcal{H}$ and let $\mathcal{H}_{\chi}$ be the corresponding quotient algebra. Define $\mathbb{H}_{\bar{\chi}}$ similarly. 
Theorem. ([L2]) $\chi \longleftrightarrow \bar{\chi}$ is a matching between real infinitesimal characters $\chi$ of $\mathcal{H}$ and infinitesimal characters $\bar{\chi}$ of $\mathbb{H}$ and

$$
\mathcal{H}_{\chi} \cong \mathbb{H}_{\bar{\chi}}
$$

We refer to section 4 in [BM2], in particular formula (4.2), and theorem 4.3 which shows that this algebra isomorphism is analytic in $(r, t)$. As a consequence, we can fix $r=1$ and transfer the study of the representation theory of $\mathcal{H}$ to $\mathbb{H}$. In order to consider unitary representations for $\mathbb{H}$, we also need a $*$ operation. This will be explained in section 2.6.

2.2. Standard Modules. Let $Z\left({ }^{L} G\right)$ denote the center of ${ }^{L} G$. For a set $\mathcal{S}$ of elements in ${ }^{L} G$ and ${ }^{L} \mathfrak{g}$, let $C_{G}(\mathcal{S})$ denote the (common) centralizer in ${ }^{L} G$ of all elements in $\mathcal{S}$, and $A_{G}(\mathcal{S})$ be the component group of $C_{G}(\mathcal{S}) . \widehat{A_{G}(\mathcal{S})}$ will denote the set of equivalent classes of irreducible representations of $A_{G}(\mathcal{S})$. When there is no confusion, we will drop the subscript $G$.

We parametrize irreducible representations of $\mathcal{H}$ as in [KL] by ${ }^{L} G$ conjugacy classes $(t, e, \psi)$, where $t \in{ }^{L} G$ is semisimple, $e \in{ }^{L} \mathfrak{g}$ is nilpotent such that $A d(t) e=$ $q e$, and certain $\left(\psi, V_{\psi}\right) \in \widehat{A(e, t)}$. The $\psi$ must satisfy the additional conditions that $\left.\psi\right|_{Z\left({ }^{L} G\right)}$ is trivial, and $\psi$ must appear in the Springer correspondence (see the details below).

Embed $e$ in a Lie triple $\{e, H, f\}$. Write $t=t_{0} t_{H}$ where $t_{H}=e^{\frac{1}{2} \log q H}$ and $t_{0}$ is a semisimple element centralizing the Lie triple. As mentioned earlier, we assume that $t_{0}$ is hyperbolic. In this case we may consider representations of $\mathbb{H}$ only. The classification of irreducible representations is then given ([L5]) by ${ }^{L} G$ conjugacy classes $\{s, e, \psi\}$ such that $s \in{ }^{L} \mathfrak{g}$ is semisimple, $e \in{ }^{L} \mathfrak{g}$ is nilpotent such that $[s, e]=e$ and $\psi \in \widehat{A(e, s)}$. In view of theorem 2.1 and the remark following it, we have assumed $\mathbf{r}$ acts by 1 .

The results in $[\mathrm{KL}]$ attach to each $\left({ }^{L} G\right.$ conjugacy class) $(e, s)$ a standard module $X(e, s)$ which decomposes under the action of $A(e, s)$ as

$$
X(e, s):=\bigoplus_{\left(\psi, V_{\psi}\right) \in \widehat{A(e, s)}} X(e, s, \psi) \otimes V_{\psi} .
$$

As a $\mathbb{C}[W]$-module,

$$
X(e, s) \cong H^{*}\left(\mathcal{B}_{e}\right),
$$

where $\mathcal{B}_{e}$ is the variety of Borel subalgebras of ${ }^{L} \mathfrak{g}$ containing $e$. The component group $A(e, s)$ is naturally a subgroup of $A(e)$ because in a connected algebraic group, the centralizer of a torus is connected. The group $A(e)$ acts on the right hand side of (2.2.2), and the action of $A(e, s)$ on the left hand side is compatible 
with the inclusion and the isomorphism. Let ${ }^{L} \mathcal{O}$ be the ${ }^{L} G$ orbit of $e$ in ${ }^{L} \mathfrak{g}$. For $\phi \in \widehat{A(e)}$, let $H^{*}\left(\mathcal{B}_{e}\right)^{\phi}$ be the $\phi$-isotypic component,

$$
H^{*}\left(\mathcal{B}_{e}\right)^{\phi}=\operatorname{Hom}_{A(e)}\left[\phi: H^{*}\left(\mathcal{B}_{e}\right)\right] .
$$

According to the Springer correspondence,

$$
H^{*}\left(\mathcal{B}_{e}\right)=\bigoplus_{\phi \in \widehat{A(e)}} H^{*}\left(\mathcal{B}_{e}\right)^{\phi} \otimes V_{\phi}
$$

Furthermore, $H^{\text {top }}\left(\mathcal{B}_{e}\right)^{\phi}$ is an irreducible representation of $W$. It is denoted $\sigma\left({ }^{L} \mathcal{O}, \phi\right)$, and each representation of $W$ is uniquely of the form $\sigma\left({ }^{L} \mathcal{O}, \phi\right)$. The correspondence is normalized so that if $e$ is the principal nilpotent element, and $\phi$ is trivial, then $\sigma\left({ }^{L} \mathcal{O}, \phi\right)=$ sgn. Comparing with (2.2.1) and (2.2.2), we conclude that

$$
\operatorname{Hom}_{W}\left[\sigma\left({ }^{L} \mathcal{O}, \phi\right): X(e, s, \psi)\right]=\left[\left.\phi\right|_{A(e, s)}: \psi\right] .
$$

Furthermore, the $\sigma\left({ }^{L} \mathcal{O}^{\prime}, \phi\right)$ occuring in $H^{*}\left(\mathcal{B}_{e}\right)$ all correspond to ${ }^{L} \mathcal{O}^{\prime}$ such that ${ }^{L} \mathcal{O} \subset \overline{L^{L} \mathcal{O}^{\prime}}$

Consider the case when $s=H / 2$, for a triple $e, H, f$. The results in [KL] imply that the modules $X(e, s, \psi)$ are irreducible, and come from the Iwahorifixed vectors of tempered representations of the p-adic group, so in particular, they are unitary. Furthermore, the multiplicity of $\sigma\left({ }^{L} \mathcal{O}, \psi\right)$ in $X(e, s, \psi)$ is 1 .

Definition. The modules $X(e, s, \psi)$ with $s=H / 2$ will be called tempered irreducible.

According to [KL], whenever $s$ is antidominant, $X(e, s, \psi)$ has a unique irreducible submodule $\bar{X}(e, s, \psi)$. When $s$ is dominant, $X(e, s, \psi)$ has a unique irreducible quotient.

The analogous formula to (2.2.4) holds whenever the data $(e, s)$ factor through a Levi component ${ }^{L} M$ :

$$
\begin{aligned}
& X(e, s)=\mathbb{H} \otimes_{\mathbb{H}_{M}} X_{M}(e, s), \\
& \mathbb{H} \otimes_{\mathbb{H}_{M}} X_{M}(e, s, \tau)=\bigoplus\left[\left.\psi\right|_{A_{M}(e, s)}: \tau\right] X(e, s, \psi)
\end{aligned}
$$

We write $\operatorname{Ind}_{M}^{G}[\pi]$ for the module $\mathbb{H} \otimes_{\mathbb{H}_{M}} \pi$.

Suppose ${ }^{L} M$ is the centralizer of $\nu$, and ${ }^{L} P={ }^{L} M^{L} N$ is such that $\langle\nu, \alpha\rangle>0$ for all roots $\alpha \in \Delta\left({ }^{L} \mathfrak{n}\right)$. Write ${ }^{L} M={ }^{L} M_{0}{ }^{L} A$, where ${ }^{L} A$ is the center. Then $A_{M}(e, s)=A_{G}(e, s)$. This is because the centralizer of $e$ is of the form $L U$ with $U$ connected unipotent, and $L$ the centralizer of $e$ and $H$. It follows that every component of $A_{G}(e, s)$ meets $L$, and therefore

$$
A_{G}(e, s)=A_{G}(e, H, \nu)=A_{M}(e, H)=A_{M}(e, s) .
$$




$$
\text { For } \begin{aligned}
\tau \in \widehat{A_{M}(e, s)}= & \widehat{A_{M_{0}}(e)}, \\
& X_{M}(e, s, \tau)=X_{M_{0}}(e, H / 2, \tau) \otimes \mathbb{C}_{\nu} .
\end{aligned}
$$

The representation $X_{M_{0}}(e, H / 2, \tau)$ is a tempered irreducible module, and if $\psi$ is the representation $\tau$ of $A_{M}(e, s)$ viewed as a representation of $A_{G}(e, s)$, then $X(e, s, \psi)=\mathbb{H} \otimes_{\mathbb{H}_{M}} X_{M}(e, s, \tau)$. The usual Langlands classification implies that $X(e, s, \psi)$ has a unique irreducible quotient. It coincides with $\bar{X}(e, s, \psi)$.

2.3. Spherical modules. We apply these ideas to the case when ${ }^{L} \mathcal{O}$ is the trivial orbit. In this case, $A(e)$ is trivial, and $X(e, s)$ is the full induced module,

$$
X(e, s)=\mathbb{H} \otimes_{\mathbb{A}} \mathbb{C}_{\nu},
$$

where $\mathbb{C}_{\nu}$ is the character of $\mathbb{A}$ corresponding to $s$ ( $\nu \in{ }^{L} \mathfrak{a}$ dominant). We abbreviate it as $X(s)$, and write $\bar{X}(s)$ for its spherical subquotient. Since $\nu$ is dominant, this coincides with the Langlands quotient. According to [BM2], $\bar{X}(s)$ is also obtained as follows. There is a unique orbit of maximal dimension, which we denote as ${ }^{L} \mathcal{O}(s)$, such that $[s, e(s)]=e(s)$ (recall that we set $r=1$ earlier) for some $e(s) \in{ }^{L} \mathcal{O}(s)$.

The Iwahori-Matsumoto involution $\mathcal{I M}$ is defined on the generators of $\mathbb{H}$ by the formulas

$$
\begin{aligned}
& \mathcal{I M}\left(t_{w}\right):=(-1)^{\ell(w)} t_{w}, \quad w \in W \\
& \mathcal{I M}(\omega):=-\omega, \quad \omega \in \mathfrak{a} .
\end{aligned}
$$

It takes $\bar{X}(s)$ to $\bar{X}(e(s), s$, triv $)$. In turn this equals $X(e(s), s$, triv $)$ because any factor would have to have as parameter an orbit of strictly larger dimension. Write again $s=H / 2+\nu^{\prime}$ where $H$ is the middle element of the triple containing $e(s)$. By the above discussion, $X(e(s), s$, triv $)$ is also induced irreducibly from $\bar{X}_{M}(e(s), s$, triv $)$, where ${ }^{L} M$ is determined by $\nu^{\prime}$ as defined right before formula (2.2.7). Applying $\mathcal{I} \mathcal{M}$, we get

$$
\bar{X}(s)=\operatorname{Ind} d_{M}^{G}\left[\bar{X}_{M}(s)\right]
$$

Unitary representations. One of the main features of Proposition 1.1 is that it implies that $\mathcal{I} \mathcal{M}$ preserves unitarity. A special case of the representations $X(e(s), s, t r i v)$ is when in fact $(s, e(s))$ can be made into a Lie triple, i.e. there is $f$ such that $[s, e(s)]=e(s),[s, f]=-f,[e(s), f]=s$. Then $X(e(s), s, \operatorname{triv})$ consists of the $\mathcal{I}$-fixed vectors of a tempered representation, therefore it is unitary. These are special cases of Arthur parameters. 
2.4. We call a face a set in the dominant chamber determined by the positive roots of ${ }^{L} G$ being $=0,=1,<1$, or $>1$. Fix such a face $\mathcal{F}$.

Theorem. The multiplicities of the $W$-types in the spherical irreducible module $\bar{X}(s)$ are constant as $s$ ranges over the face $\mathcal{F}$.

Proof. The orbit ${ }^{L} \mathcal{O}(s)$ can be characterized in the following way. Let

$$
{ }^{L} \mathfrak{g}_{i}:=\left\{X \in{ }^{L} \mathfrak{g}:[s, X]=i X\right\} .
$$

Then ${ }^{L} \mathcal{O}(s)$ is the unique orbit such that ${ }^{L} \mathcal{O}(s) \cap{ }^{L} \mathfrak{g}_{1}$ is dense in ${ }^{L} \mathfrak{g}_{1}$ ([BM2]). This intersection is also a single orbit under $C_{G}(s)$, the centralizer of $s$ in ${ }^{L} G$. The set of roots equal to zero, and equal to one, on $s$ do not change as $s$ varies over the face $\mathcal{F}$. Thus the orbit ${ }^{L} \mathcal{O}(s)$ does not change, nor does $C_{G}(s)$. Up to conjugation, we can write the parameter as $s=H / 2+\nu$, and $\bar{X}(s)=\operatorname{In} d_{M}^{G}\left(\sigma \otimes \mathbb{C}_{\nu}\right)$. Here $\sigma$ is anti-tempered, i.e. it is $\mathcal{I} \mathcal{M}$ of a tempered module. In fact $\mathcal{I} \mathcal{M}(\sigma)$ has (KazhdanLusztig) parameter $(e, H / 2$, triv $)$. Up to conjugation there are only finitely many possible $\left({ }^{L} M, \sigma\right)$.

Fix such a data, and let $\nu$ range over the center of ${ }^{L} M$. The only way that the W-structure of $\bar{X}(s)$ can change is if $A_{G}(e, s)$ differs from $A_{M}(e, s)$. Consider the centralizer $C_{G}(e)$. The one parameter group $\exp (t H)$ acts on $C_{G}(e)$, and by [BV], proposition 2.4, its component group coincides with the component group of $C_{G}(e, H)$. But $s$ commutes with $H$ and stabilizes $C_{G}(e)$. The fixed points of $s$ in $C_{G}(e)$ also have an action of $\exp (t H)$, and the argument in [BV] proposition 2.4, shows that all the components meet the centralizer of $H$ in $C_{G}(e, s)$, which is also the centralizer of $\nu$ in $C_{G}(e, s)$. Thus every component of $C_{G}(e, s)$ meets $C_{M}(e, s)$, so the only way the multiplicities of $\bar{X}(s)$ can change is if the centralizer of $\nu$ becomes larger.

Let $\nu_{0}$ be such a point, and assume there are $\nu_{i} \rightarrow \nu_{0}$ such that $H / 2+\nu_{i}$ are conjugate by a single $w \in W$ to points in $\mathcal{F}$ for all $i \geq 0$. Assume that the centralizer in ${ }^{L} \mathfrak{g}$ of all the $\nu_{i}$ for $i>0$ is $\mathfrak{m}^{\prime}$, while the centralizer of $\nu_{0}$ is $\mathfrak{m}\left(\nu_{0}\right)$. Then $\mathfrak{m}^{\prime} \varsubsetneqq \mathfrak{m}\left(\nu_{0}\right)$, is a Levi component. Since the zero subspace of $s$ cannot change in $\mathcal{F}, H$ cannot have any kernel on $\mathfrak{m}\left(\nu_{0}\right)$. There is a decomposition $\mathfrak{m}\left(\nu_{0}\right)=\mathfrak{n}^{\prime}+\mathfrak{m}^{\prime}+\overline{\mathfrak{n}^{\prime}}$. The Lie triple is in $\mathfrak{m}^{\prime}$, so the corresponding $\operatorname{sl}(2)$ acts on both $\mathfrak{n}^{\prime}, \overline{\mathfrak{n}^{\prime}}$. Since it has no eigenvalues of zero, all the eigenvalues of $H / 2$ are half-integers. But the component group $A(e)$ in any semisimple Lie algebra $\mathfrak{l}$ is the same as the component group in $A(e, H)$, so it is the same as $A(e, \exp (i \pi H))$. The Lie algebra of the centralizer of $\exp (i \pi H)$ is formed of even eigenspaces of $H$ only. Applied to $\mathfrak{l}=\mathfrak{m}\left(\nu_{0}\right)$, this implies that the component group with respect to $M^{\prime}$ and $M\left(\nu_{0}\right)$ are the same.

The argument implies that a face $\mathcal{F}$ is a finite union of closed (in $\mathcal{F}$ ) sets on which the $W$-structure of $\bar{X}(s)$ does not change. Since $\mathcal{F}$ is connected, these 
sets cannot have empty intersection, and this implies that the $W$ multiplicities in $\bar{X}(s)$ are constant for $s$ over $\mathcal{F}$.

2.5. Unequal Parameters. We consider the following modification of the graded Iwahori-Hecke algebra 2.1. Let

$$
L: R \longrightarrow \mathbb{R}^{+}
$$

be a function which is constant on $\mathrm{W}$-orbits, and write

$$
c_{\alpha}:=L(\alpha) \text {. }
$$

Then replace relations (2.1.6) by

$$
\begin{aligned}
t_{w} t_{w^{\prime}} & =t_{w w^{\prime}}, \\
t_{s}^{2} & =1, \\
t_{s} \omega & =s(\omega) t_{s}+c_{\alpha}\langle\omega, \check{\alpha}\rangle, \quad s=s_{\alpha}, \omega \in \mathfrak{a} .
\end{aligned}
$$

Many, but not all, of the results for the case $c_{\alpha}=1$ extend to this situation (cf. [L4]-L7). In particular, except when mentioned explicitly, we will not use any results established only for $c_{\alpha}=1$.

2.6. Hermitian Modules. The * operation also transfers to the graded version. We refer to $\S 5$ of [BM2] for the details. Here is a summary of what we need. Let $w_{0} \in W$ be the longest element and $t_{0}$ be the corresponding element in $\mathbb{C}[W]$. Since $\mathfrak{a}=\mathcal{X} \otimes_{\mathbb{Z}} \mathbb{C}$, it has a conjugation coming from the complex conjugation on $\mathbb{C}$. We denote it by ${ }^{-}$. Let $\iota(\omega)=(-1)^{\operatorname{deg} \omega} \bar{\omega}$ and $\tilde{\omega}=w_{0} \iota(\omega)$.

Theorem. (§5 in $[\mathrm{BM} 2])$ Let $\omega \in \mathbb{A}$. Then

$$
\begin{aligned}
t_{w}^{*} & =t_{w^{-1}}, \\
\omega^{*} & =t_{0} \cdot \tilde{\omega} \cdot t_{0} .
\end{aligned}
$$

In particular, if $\omega \in \mathfrak{a}$, then

$$
\omega^{*}=-\bar{\omega}+\sum_{\beta \in R^{+}} c_{\beta}\langle\bar{\omega}, \check{\beta}\rangle t_{s_{\beta}},
$$

where $s_{\beta} \in W$ is the reflection about $\beta$.

In the case when $c_{\alpha}=1$, recall that $(e, H, f)$ is a Lie triple corresponding to $e$. Write $s=s_{0}+s_{H}$, and recall that $s_{0}$ is assumed to be hyperbolic. An irreducible representation admits a Hermitian form if and only if $(s, e, \psi)$ is conjugate to $\left(s^{\prime}=\overline{s_{0}} s_{H}, e, \psi\right)$ (theorem 5.2 in [BM2]).

Fix a (standard) parabolic subgroup ${ }^{L} P$ with Levi decomposition ${ }^{L} P={ }^{L} M^{L} N$. Let $\mathbb{H}_{M}$ be the corresponding (graded) Hecke subalgebra (with roots $R_{M} \subset R$ ) 
and let $W(M) \subset W$ be the corresponding Weyl group. Every element $a \in \mathbb{H}$ can be written uniquely as

$$
a=\sum_{w \in W / W(M)} t_{w} m_{w}
$$

with $m_{w} \in \mathbb{H}_{M}$. Thus there is a well defined map

$$
\epsilon_{M}: \mathbb{H} \longrightarrow \mathbb{H}_{M}, \quad \epsilon_{M}(a):=m_{1}, \text { the component of } a \text { in } \mathbb{H}_{M} .
$$

Recall the Bruhat order on the elements of $W$. Write $w^{\prime} \rightarrow w$ if there exists a reflection $s_{\alpha}$, for some root $\alpha$, such that $w=w^{\prime} s_{\alpha}$ and $\ell(w)>\ell\left(w^{\prime}\right)$. Define $w^{\prime}<w$ if there is a sequence $w^{\prime}=w_{1} \rightarrow w_{2} \rightarrow \cdots \rightarrow w_{p}=w$.

Proposition. Denote by $*_{M}$ the map corresponding to the star operation $*_{M}$ : $a \mapsto a^{*}$ in $\mathbb{H}_{M}$, and denote by $*_{G}$ the corresponding map in $\mathbb{H}$. Then

$$
\epsilon_{M}\left(*_{G} a\right)=*_{M} \epsilon_{M}(a) \quad \text { for all } a \in \mathbb{H} \text {. }
$$

Proof. First observe that

$$
\epsilon_{M}\left(t_{m_{1}} a t_{m_{2}}\right)=t_{m_{1}} \epsilon_{M}(a) t_{m_{2}}, \quad \text { for } \quad m_{i} \in W(M) .
$$

Let $\omega \in \mathbb{A}$. The longest element $w_{0}$ decomposes as $w_{0}=w_{l} w_{0}^{M}$, where $w_{0}^{M}$ is the longest element in $W(M) . w_{l}$ has the property that it is a minimal element (in the Bruhat order) in $w_{0} W(M)$. Let $t_{0}, t_{l}$ and $t_{0}^{M}$ be the corresponding elements in $\mathbb{H}$. Since $t_{0}^{-1}=t_{0}$ and $\left(t_{0}^{M}\right)^{-1}=t_{0}^{M}$, the relation

$$
t_{0}=t_{l} t_{0}^{M}=t_{0}^{M} t_{l}^{-1}
$$

holds. Then we get

$$
*_{G}(\omega)=t_{0} \cdot \tilde{\omega} \cdot t_{0}=t_{l} t_{0}^{M} \cdot w_{0}^{M} w_{l}^{-1}(\iota(\omega)) \cdot t_{l} t_{0}^{M} .
$$

On the other hand,

$$
w_{0}^{M} w_{l}^{-1} \iota(\omega) \cdot t_{l}=t_{l} \cdot w_{l}^{-1} w_{0}^{M} w_{l}^{-1} \iota(\omega)+\sum_{x<w_{l}} t_{x} \omega_{x}=t_{l} \cdot w_{0}^{M} \iota(\omega)+\sum_{x<w_{l}} t_{x} \omega_{x}
$$

where the sum ranges over $x \in W$ which are smaller than $w_{l}$ in the Bruhat order. Combining this with (2.6.6), and the formula for $*_{M}$ in theorem 2.6, we get

$$
*_{G}(\omega)=*_{M}(\omega)+\sum_{x<w_{l}} t_{l} t_{0}^{M} t_{x} \omega_{x} t_{0}^{M} .
$$

Therefore, using 2.6.4,

$$
\epsilon\left(*_{G}(\omega)\right)=*_{M}(\omega)+t_{0}^{M} \epsilon\left(t_{l}^{-1} t_{x}\right) \omega_{x} t_{0}^{M}=*_{M}(\omega)+t_{0}^{M} \epsilon\left(t_{w_{l}^{-1} x}\right) \omega_{x} t_{0}^{M} .
$$

To prove the formula in the proposition for $a=\omega$, we must show that $\epsilon\left(t_{w_{l}^{-1} x}\right)=$ 0 for $x<w_{l}$. But if $w_{l}^{-1} x=m \in W(M)$, then $w_{l}=x m^{-1}$ with $x<w_{l}$, contradicting the minimality property of $w_{l}$. 
Now consider an element $t_{w} \omega$ such that $w \notin W(M)$. Then $\epsilon_{M}\left(t_{w} \omega\right)=0$. We need to show that $\epsilon_{M}\left(*_{G}(\omega) t_{w^{-1}}\right)=0$ as well. For this, write $*_{G}(\omega)=t_{0} \cdot \tilde{\omega} \cdot t_{0}$ and decompose

$$
t_{0} \cdot \tilde{\omega} \cdot t_{0} \cdot t_{w^{-1}}=t_{0} \cdot \tilde{\omega} \cdot t_{w_{0} w^{-1}}=\sum_{x<w_{0} w^{-1}} t_{w_{0} x} \omega_{x}, \quad \omega_{x} \in \mathbb{A} .
$$

To prove the claim we need to show $w_{0} x \notin W(M)$. Suppose $w_{0} x=m$. Then $x=w_{0} m$, and so $w_{0} m<w_{0} w^{-1}$. It follows that $w_{0} w^{-1} \in w_{0} W(M)$, because $w_{0}$ is the long element. But this implies $w^{-1} \in W(M)$, a contradiction. Thus $\epsilon_{M}\left(*_{G}(\omega) t_{w^{-1}}\right)=0$ as claimed.

If $\mathcal{W}$ is a module for $\mathbb{H}_{M}$, then we can form the induced module

$$
\operatorname{Ind} d_{M}^{G}(\mathcal{W}):=\mathbb{H} \otimes_{\mathbb{H}_{M}} \mathcal{W} .
$$

This has a basis $\left\{t_{x} \otimes v\right\}$ where $v \in \mathcal{W}$ and $x \in W / W(M)$. We will denote by $\mathcal{W}^{h}$ the Hermitian dual of the module $\mathcal{W}$.

Corollary. The Hermitian dual of $\operatorname{Ind}_{M}^{G}(\mathcal{W})$ is $\operatorname{Ind}_{M}^{G}\left(\mathcal{W}^{h}\right)=\mathbb{H} \otimes_{\mathbb{H}_{M}} \mathcal{W}^{h}$. More precisely, let $t_{x} \otimes v_{x} \in \operatorname{Ind} d_{M}^{G}(\mathcal{W}), t_{y} \otimes v_{y} \in \operatorname{Ind} d_{M}^{G}\left(\mathcal{W}^{h}\right)$, and $\langle,\rangle_{M}$ be the pairing of $\mathcal{W}$ with $\mathcal{W}^{h}$. Then the pairing between $\operatorname{Ind}_{M}^{G}(\mathcal{W})$ and $\operatorname{Ind} d_{M}^{G}\left(\mathcal{W}^{h}\right)$ is given by

$$
\left\langle t_{x} \otimes v_{x}, t_{y} \otimes v_{y}\right\rangle:=\left\langle\epsilon_{M}\left(t_{y}^{*} t_{x}\right) v_{x}, v_{y}\right\rangle_{M} .
$$

Proof. First observe that these spaces are finite dimensional, so it is sufficient to construct an injection

$$
\mathbb{H} \otimes_{\mathbb{H}_{M}} \mathcal{W}^{h} \longrightarrow\left[\mathbb{H} \otimes_{\mathbb{H}_{M}} \mathcal{W}\right]^{h} .
$$

The pairing $\langle$,$\rangle gives such an injection with the required properties by propo-$ sition 2.6.

2.7. Langlands Classification. In order to simplify the notation, we will drop the superscript $L$ in the notation of Levi components $M$. Note however that all Levi components are still in the dual group. The reference for the Langlands classification, in the context of graded Hecke algebras, is [E]. See also [KR] for additional results.

Suppose $a$ is an isomorphism between two root data $\mathcal{R}_{1}$ and $\mathcal{R}_{2}$. This induces an isomorphism of the corresponding Hecke algebras,

$$
a: \mathbb{H}_{2} \longrightarrow \mathbb{H}_{1} \text {. }
$$

In particular, let $M_{1}, M_{2}$ be two Levi components of standard parabolic subgroups. Suppose $w \in W$ satisfies $w M_{1} w^{-1}=M_{2}$ and is minimal in its double coset $W\left(M_{2}\right) w W\left(M_{1}\right)$. Then the above discussion gives an isomorphism

$$
a_{w}: \mathbb{H}_{M_{2}} \longrightarrow \mathbb{H}_{M_{1}}
$$

which we use to transfer representations $\mathcal{V}$ of $\mathbb{H}_{M_{1}}$ to $w \mathcal{V}$ of $\mathbb{H}_{M_{2}}$. 
If $V$ is a (finite dimensional) irreducible $\mathbb{H}$-module, then $V$ has a generalized weight space decomposition with respect to the abelian subalgebra $\mathbb{A}$ :

$$
V=\bigoplus_{\lambda \in L_{\mathfrak{a}}} V_{\lambda}
$$

The set of $\lambda$ in the decomposition are the weights of $V$. Let $\omega_{i} \in \mathfrak{a}$ denote the fundamental weight corresponding to the simple coroot $\check{\alpha}_{i} \in{ }^{L} \mathfrak{a}$.

Definition. Let $V$ be an irreducible $\mathbb{H}$-module. $V$ is called tempered if all its weights $\lambda$ have the property that $\left\langle R e \lambda, \omega_{i}\right\rangle \leq 0$, for all fundamental weights $\omega_{i}$.

It follows from the definition that, if $\mathcal{W}$ is a tempered module, then $\mathcal{W}^{h}$ is also tempered.

For $c_{\alpha}=1$, definition 2.7 agrees with definition 2.2 ([KL]). In [L6], Lusztig gives a geometric classification of the tempered $\mathbb{H}$-modules for some particular families of (unequal) parameters $c_{\alpha}$, which correspond to unipotent representations of split p-adic groups ([L4],[L5],[L7]). In these cases, the situation is the same as for equal parameters. For arbitrary parameters $c_{\alpha}$, the results needed about the unitarity of tempered representations are still lacking. See [O] for results in this direction.

According to the (classical version of the) Langlands classification, every irreducible module can be realized as the unique irreducible quotient $L(M, \mathcal{W}, \nu)$ of a standard module

$$
X(M, \mathcal{W}, \nu):=\mathbb{H} \otimes_{\mathbb{H}_{M}}\left[\mathcal{W} \otimes \mathbb{1}_{\nu}\right],
$$

where

$\mathcal{W}$ is tempered irreducible (definition 2.7) and

$$
\langle\text { Re } \nu, \alpha\rangle>0 \text {, for all } \alpha \in R^{+}-R_{M}^{+}
$$

The module $X(M, \mathcal{W}, \nu)$ of course coincides with $\operatorname{Ind} d_{M}^{G}\left(\mathcal{W} \otimes \mathbb{1}_{\nu}\right)$, but we use the notation (2.7.4) to emphasize that it satisfies (2.7.5). Two Langlands quotients $L(M, \mathcal{W}, \nu)$ and $L\left(M^{\prime}, \mathcal{W}^{\prime}, \nu^{\prime}\right)$ are isomorphic if and only if there is $w \in W$ such that

$$
w(M, \mathcal{W}, \nu)=\left(M^{\prime}, \mathcal{W}^{\prime}, \nu^{\prime}\right) .
$$

If on the other hand $\langle\operatorname{Re} \nu, \alpha\rangle<0$, then the module $\operatorname{Ind}\left(\mathcal{M}\left(\mathcal{W} \otimes \mathbb{1}_{\nu}\right)\right.$ has a unique irreducible submodule, namely $L\left(w_{m} M, w_{m} \mathcal{W}, w_{m} \nu\right)$, where $w_{m}$ is the minimal element in $W\left(w_{0} M\right) w_{0} W(M)$.

In the case $c_{\alpha}=1$, the module $X(M, \mathcal{W}, \nu)$ corresponds to data $(e, s, \psi)$, where $(e, \psi)$ determine the tempered representation $\mathcal{W}$ (see section 2.2), and $s$ can be written as $s=s_{M}+\nu$, where $s_{M}$ is the tempered part of the parameter. A factor of $X(M, \mathcal{W}, \nu)$ has Langlands parameter $\left(M^{\prime}, \mathcal{W}^{\prime}, \nu^{\prime}\right)$ with $\left\|\nu^{\prime}\right\| \leq\|\nu\|$, 
with equality if and only if $\left(M^{\prime}, \mathcal{W}^{\prime}, \nu^{\prime}\right)=(M, \mathcal{W}, \nu)$. This follows from lemma 2.8. in chapter XI of [BW]. Therefore, all factors of an $X(e, s, \psi)$ other than $\bar{X}(e, s, \psi)$ correspond to ${ }^{L} \mathcal{O}^{\prime}$ satisfying

$$
\overline{{ }^{L} \mathcal{O}^{\prime}} \supset{ }^{L} \mathcal{O},{ }^{L} \mathcal{O}^{\prime} \neq{ }^{L} \mathcal{O}
$$

In particular, in the notation of section $2.1, \bar{X}(e, s, \psi)$ is characterized by the fact that it contains the Weyl group representation $\sigma\left({ }^{L} \mathcal{O}, \phi\right)$ with multiplicity $\left[\left.\phi\right|_{A(e, s)}: \psi\right]$.

Proposition. The Hermitian dual of $L(M, \mathcal{W}, \nu)$ is $L\left(w_{0} M, w_{m} \mathcal{W}^{h},-w_{0} \bar{\nu}\right)$. In particular, $L$ is Hermitian if and only if there is an element $w$ such that

$$
w \cdot(M, \mathcal{W}, \nu)=\left(M, \mathcal{W}^{h},-\bar{\nu}\right) .
$$

Proof. The Hermitian dual of $X(M, \mathcal{W}, \nu)$ is $\operatorname{Ind} d_{M}^{G}\left(\mathcal{W}^{h} \otimes \mathbb{1}_{-\bar{\nu}}\right)$. This is not a standard module because $-\bar{\nu}$ fails to satisfy (2.7.5). However $\langle-\operatorname{Re} \bar{\nu}, \check{\alpha}\rangle<0$, so $\operatorname{Ind}_{M}^{G}\left(\mathcal{W}^{h} \otimes \mathbb{1}_{-\bar{\nu}}\right)$ has $L\left(w_{0} M, w_{m} \mathcal{W}^{h},-w_{0} \bar{\nu}\right)$ as unique irreducible submodule. Thus

as claimed.

$$
L(M, \mathcal{W}, \nu)^{h} \cong L\left(w_{0} M, w_{m} \mathcal{W}^{h},-w_{0} \bar{\nu}\right)
$$

For $c_{\alpha}=1$, all tempered irreducible modules are known to be unitary, and therefore Hermitian, so the Hermitian condition in the proposition becomes $w$. $(M, \mathcal{W}, \nu)=(M, \mathcal{W},-\bar{\nu})$. For arbitrary $c_{\alpha}$, we will only consider, in this paper, the case of spherical modules with real infinitesimal character. In the Langlands classification, they correspond to $M=A$ (the maximal split torus), $\mathcal{W}=$ Triv, and $\nu$ dominant. Thus, the condition in proposition 2.7 is equivalent to the requirement that $w \nu=-\nu$, for some Weyl element $w$. In types $\mathrm{B} / \mathrm{C}$ (and $\nu$ real dominant), $w_{0} \nu=-\nu$, and therefore all spherical modules are Hermitian.

2.8. Intertwining operators. We use the notation of sections 2.6 and 2.7 . Suppose that $w M_{1} w^{-1}=M_{2}$ is minimal in its double coset $W\left(M_{2}\right) w W\left(M_{1}\right)$ (or rather the ${ }^{L} M$ 's). Given a simple reflection $s_{\alpha}$, we can form the element $r_{\alpha} \in \mathbb{H}$,

$$
r_{\alpha}:=t_{s_{\alpha}} \alpha-c_{\alpha}
$$

Lemma. The elements $r_{\alpha}$ satisfy $\omega r_{\alpha}=r_{\alpha} s(\omega)$. Let $w=s_{\alpha_{1}} \cdots \cdots s_{\alpha_{k}}$ be a reduced decomposition. Then $r_{w}=\prod r_{\alpha_{i}}$ does not depend on the reduced decomposition of $w$.

Proof. The first relation is a simple application of the defining relations for $\mathbb{H}$ :

$$
\begin{aligned}
& \omega\left(t_{s_{\alpha}} \alpha-c_{\alpha}\right)=\left(t_{s_{\alpha}} s(\omega)+c_{\alpha}\langle\omega, \check{\alpha}\rangle\right) \alpha-c_{\alpha} \omega= \\
& \quad=\left(t_{s_{\alpha}} \alpha-c_{\alpha}\right) s(\omega)+c_{\alpha}(s(\omega)-\omega)+c_{\alpha}\langle\omega, \check{\alpha}\rangle \alpha=\left(t_{s_{\alpha}} \alpha-c_{\alpha}\right) s(\omega) .
\end{aligned}
$$


More generally if $f$ is a rational function in one variable, then

$$
f(\omega) r_{\alpha}=r_{\alpha} f\left(s_{\alpha} \omega\right) .
$$

Recall from [L2] (proposition 5.2) that

$$
\bar{\tau}_{\alpha}=t_{s_{\alpha}} \frac{\alpha}{\alpha+c_{\alpha}}-\frac{c_{\alpha}}{\alpha+c_{\alpha}}=\frac{1}{-\alpha+c_{\alpha}} r_{\alpha}
$$

and that $\bar{\tau}_{w}=\prod \bar{\tau}_{i}$ is well defined (independent of the reduced decomposition). Write

$$
R_{w}=\left\{\alpha_{1}, s_{1} \alpha_{2}, \ldots, s_{1} s_{2} \ldots s_{k-1} \alpha_{k}\right\}=\left\{\beta>0: w^{-1} \beta<0\right\} .
$$

Then substituting (2.8.3) for each $r_{i}$ and applying (2.8.2) repeatedly we get

$$
\prod r_{i}=\prod\left(-\alpha_{i}+c_{\alpha}\right) \bar{\tau}_{i}=\left(\prod_{\beta \in R_{w}}\left(-\beta+c_{\alpha}\right)\right) \prod \bar{\tau}_{i}=\left(\prod_{\beta \in R_{w}}\left(-\beta+c_{\alpha}\right)\right) \bar{\tau}_{w}
$$

The claim follows.

Proposition. Let $\mathcal{V}$ be a representation of $\mathbb{H}_{M_{1}}$. Then the operator $A_{w}: \operatorname{Ind}_{M_{1}}^{G}(\mathcal{V}) \longrightarrow$ $\operatorname{Ind}_{M_{2}}^{G}(w \mathcal{V})$ defined by

$$
A_{w}\left(t_{x} \otimes_{\mathbb{H}_{M_{1}}} v\right):=t_{x} r_{w} \otimes_{\mathbb{H}_{M_{2}}} v
$$

is an intertwining operator.

Proof. We need to check that $A_{w}$ is well defined, i.e. it satisfies

$$
\omega r_{w}=r_{w} w^{-1}(\omega), \quad t_{s_{\alpha}} r_{w}=r_{w} t_{w^{-1} \alpha}, \quad \alpha \in \Delta\left(M_{1}\right) \text { a simple root. }
$$

The first relation follows from a repeated application of lemma 2.8. For the second relation, $r_{\alpha} r_{w}=r_{w} r_{w^{-1} \alpha}$ holds. We verify that $\alpha r_{w}=r_{w} w^{-1} \alpha$. It is sufficient to check for $w=s_{\beta}$, a simple reflection.

$$
\begin{aligned}
& \alpha\left(t_{s_{\beta}} \beta-c_{\alpha}\right)=\alpha t_{s_{\beta}} \beta-c_{\alpha} \alpha=\left(t_{s_{\beta}} s_{\beta}(\alpha)+c_{\alpha}\langle\check{\beta}, \alpha\rangle\right) \beta-c_{\alpha} \alpha \\
& \quad=t_{s_{\beta}} \beta s_{\beta}(\alpha)-c_{\alpha}(\alpha-\langle\check{\beta}, \alpha\rangle \beta)=\left(t_{s_{\beta}} \beta-c_{\alpha}\right) s_{\beta}(\alpha) .
\end{aligned}
$$

Substituting $r_{\alpha}=t_{s_{\alpha}} \alpha-c_{\alpha}$ and using $\alpha r_{w}=r_{w} w^{-1} \alpha$, we get the second relation. The fact that the map is an intertwining operator follows from the nature of the action of $\mathbb{H}$ which is by multiplication on the left. 
2.9. We apply the results in 2.8 to the Langlands classification. Recall $w_{m}$ the minimal element in the double coset $W(M) w_{0} W\left(w_{0} M\right)$. Then multiplication on the right by $r_{m}:=r_{w_{m}}$ is an intertwining operator

$$
A_{m}: X(M, \mathcal{W}, \nu) \longrightarrow \operatorname{Ind}_{w_{0} M}^{G}\left(w_{m} \mathcal{W} \otimes \mathbb{1}_{w_{0} \nu}\right)
$$

This is identically zero precisely when $r_{m} \otimes\left[v \mathbb{1}_{\nu}\right]=0$ for all $v \in \mathcal{W}$. When it is not identically zero, the image is precisely $L(M, \mathcal{W}, \nu)$ because this submodule is generated by any vector whose generalized eigenvalue under $\mathbb{A}$ projects onto $\nu$. On the other hand, observe that the leading term of $r_{m}$ is $t_{m} \prod_{(\alpha, \nu)>0} \alpha$. But

$$
\left(\prod_{(\alpha, \nu)>0} \alpha\right) \cdot v \mathbb{1}_{\nu}=\prod_{(\alpha, \nu)>0}(\alpha, \nu+\chi) v \mathbb{1}_{\nu}
$$

where $\chi$ is the infinitesimal character of $\mathcal{W}$. This is because $\prod_{(\alpha, \nu)>0} \alpha$ is invariant under $W(M)$ therefore in the center of $\mathbb{H}_{M}$. Thus $A_{m}$ is not identically zero as a function of $\nu$. For a full principal series (2.9.2) implies that $A_{m}$ is not identically zero for any $\nu$ satisfying (2.7.5). But for more general induced representations $A_{m}$ could be zero for certain values of $\nu$. On the other hand, it follows from the theory of intertwining operators on the p-adic group, that there exists a family of intertwining operators $B_{m}(\nu)$

$$
B_{m}: X(M, \mathcal{W}, \nu) \longrightarrow \operatorname{Ind}_{w_{m} M}^{G}\left(w_{m} \mathcal{W} \otimes \mathbb{1} w_{m} \nu\right)
$$

which is analytic as a function of $\nu$ and whose image is $L(M, \mathcal{W}, \nu)$ for all $\nu$ satisfying (2.7.5). We claim that there is a meromorphic function $f(\nu)$ such that $f(\nu) A_{m}=B_{m}(\nu)$. This goes as follows. Recall (section 2.2) that $X$ has a lowest K-type $\mu$ occuring with multiplicity 1 . Then there are analytic functions $g$ and $h$ such that for any vector $v \in X$ transforming according to $\mu$, we have $A_{m}(v)=g(\nu) v$ and $B_{m} v=h(\nu) v$. Then $f=h g^{-1}$.

We will not need this refinement.

2.10. Suppose $\mathcal{W}$ is Hermitian tempered, and that $L(M, \mathcal{W}, \nu)$ is Hermitian with $\nu$ real. Let $w$ be such that $w(M, \mathcal{W}, \nu)=(M, \mathcal{W},-\nu)$, minimal in its double coset $W(M) w W(M)$. This double coset is also $W(M) w_{0} W(M)$. This is because both $w \nu$ and $w_{0} \nu$ are antidominant (so $w_{0} \nu=w \nu=-\nu$ ) and $R_{M}=\{\alpha \in R$ : $\langle\alpha, \nu\rangle=0\}$. Recall the isomorphism $a_{w}$ from (2.7.2), and denote by $\tau: \mathcal{W} \longrightarrow \mathcal{W}$ an isomorphism (unique up to a scalar) satisfying $\tau(h \cdot v)=a_{w}(h) \cdot \tau(v)$. Recall that we can decompose $M={ }^{0} M \cdot A$, where $A$ is the (split part of the) center of $M$. Then we can write any element $m \in \mathbb{H}_{M}$ as $m=\sum m_{i} a_{i}$ with $m_{i} \in \mathbb{H}{ }^{{ }_{0}} M$ and $a_{i} \in A$. Thus it makes sense to evaluate $m \in \mathbb{H}_{M}$ at $\nu$. We write this as $m(\nu)$. With this notation

$$
\sum m_{i} a_{i}\left(v \mathbb{1}_{\nu}\right)=\sum m_{i} a_{i}(\nu) v \mathbb{1}_{v}=(m(\nu) v) \mathbb{1}_{\nu} .
$$


Corollary. Suppose $\mathcal{W}$ is Hermitian tempered and that $X(M, \mathcal{W}, \nu)$ is Hermitian irreducible with $\nu$ is real. Then up to a nonzero scalar the inner product is given by the formula

$$
\left\langle t_{x} \otimes v_{x} \mathbb{1}_{\nu}, t_{y} \otimes v_{y} \mathbb{1}_{\nu}\right\rangle=\left\langle\epsilon\left(t_{y}^{*} t_{x} r_{w}\right)(\nu) \tau\left(v_{x}\right), v_{y}\right\rangle_{M} .
$$

Proof. This follows from sections 2.3-2.9.

2.11. Let $M$ be a proper Levi component, and $\sigma$ be a tempered module of $\mathbb{H}_{M}$. Assume that $\nu$ is such that $\langle\nu, \gamma\rangle=0$ for $\gamma \in R_{M}$, but $\langle\nu, \alpha\rangle>0$ for some simple root $\alpha$. This is always the case, unless $\nu$ is antidominant. Let $M_{\alpha}$ be the Levi component generated by $M$ and the root vectors corresponding to $\pm \alpha$. Then there is a shortest Weyl group element $w_{\alpha} \in W\left(M_{\alpha}\right)$ so that $w_{\alpha} \nu$ is nonpositive on the roots in $R_{M_{\alpha}}$. By using a reduced decomposition for $w_{\alpha}$, we can construct an intertwining operator

$$
A_{\alpha}(\nu): \mathbb{H} \otimes_{M} \sigma \mathbb{1}_{\nu} \longrightarrow \mathbb{H} \otimes_{w_{\alpha}(M)} w_{\alpha}(\sigma) \mathbb{1}_{w_{\alpha}(\nu)},
$$

which is induced from the corresponding intertwining operator for $\mathbb{H}_{M_{\alpha}}$. The data $\left(w_{\alpha}(M), w_{\alpha}(\sigma)\right)$ are similar to $(M, \sigma)$. But $w_{\alpha}(\nu)$ is positive on fewer roots than $\nu$. Furthermore, $M$ and $w_{\alpha}(M)$ are Levi components of maximal parabolic subgroups of $M_{\alpha}$.

Apply this idea repeatedly to a Langlands parameter $(M, \sigma, \nu)$ (with $\nu$ positive on roots not in $R_{M}$ ). We find that the element $w_{m}$ (notation as in section 2.9) decomposes into

$$
w_{m}=\prod w_{\alpha_{i}}, \quad \ell\left(w_{m}\right)=\sum \ell\left(w_{\alpha_{i}}\right) .
$$

As before, write $w_{i}=w_{\alpha_{k-i+1}} \ldots w_{\alpha_{k}}$. The intertwining operator $A_{m}$ decomposes accordingly into a product

$$
A_{m}(\nu)=\prod A_{i}\left(w_{i}(M), w_{i}(\sigma), w_{i}(\nu)\right) .
$$

Each $A_{i}$ is induced from a similar operator on a Levi component, and there are no poles when $(M, \sigma, \nu)$ is the parameter of a standard module.

We will use this decomposition in the spherical case, when $\sigma$ is the $\mathcal{I M}$ of a tempered module.

2.12. We specialize to the spherical case. With notation as in $2.10, M=A$, $\mathcal{W}=$ Triv, and $w_{0}$ the long Weyl group element. Let $\nu \in{ }^{L} \mathfrak{a}$ and assume that $w_{0} \nu=-\nu$. Formula (2.9.1) becomes

$$
A(\nu): X(\nu) \longrightarrow \operatorname{Ind}_{A}^{G}\left(\mathbb{1}_{w_{0} \nu}\right), \quad x \otimes \mathbb{1}_{\nu} \mapsto x r_{w_{0}} \otimes \mathbb{1}_{-\nu} .
$$

If $w_{0}=s_{\alpha_{1}} \ldots s_{\alpha_{k}}$ is a reduced decomposition, let $w_{i}=s_{\alpha_{k-i+1}} \ldots s_{\alpha_{k}}$. Then write

$$
r_{w_{0}}(\nu)=\prod\left(-t_{s_{\alpha_{i}}}\left\langle\alpha_{i}, w_{i} \nu\right\rangle-c_{\alpha}\right) .
$$


The operator $A(\nu)$ is rewritten as

$$
x \otimes \mathbb{1}_{\nu} \mapsto x r_{w_{0}}(\nu) \otimes \mathbb{1}_{-\nu} .
$$

Taking into account that $X(\nu) \cong \mathbb{C}[W]=\sum V_{\sigma} \otimes V_{\sigma^{*}}, A(\nu)$ induces an operator

$$
a(\sigma, \nu): V_{\sigma^{*}} \longrightarrow V_{\sigma^{*}}, \quad \prod\left(-\sigma^{*}\left(t_{s_{\alpha_{i}}}\right)\left\langle\alpha_{i}, w_{i} \nu\right\rangle-c_{\alpha}\right) .
$$

When $\sigma=$ Triv, this operator is the scalar

$$
\prod\left(-\left\langle\alpha_{i}, \nu\right\rangle-c_{\alpha}\right)
$$

This scalar is never zero if $\nu$ is dominant. We divide $A(\nu)$ by it. This new operator gives rise to an $a(\sigma, \nu)$ with the property that $a(\operatorname{triv}, \nu)=I d$. More general, for any $i$ and any $\nu$, define $a_{i}(\sigma, \nu)$ by the relations

$$
a_{i}(\sigma, \nu)= \begin{cases}1 & \text { on the }(+1) \text {-eigenspace of } s_{\alpha_{i}} \text { on } \sigma^{*} \\ \frac{c_{\alpha}-\left\langle\alpha_{i}, \nu\right\rangle}{c_{\alpha}+\left\langle\alpha_{i}, \nu\right\rangle} & \text { on the }(-1) \text {-eigenspace of } s_{\alpha_{i}} \text { on } \sigma^{*}\end{cases}
$$

If $w=s_{1} \ldots s_{k}$ is a reduced decomposition, and $w_{i}=s_{k-i+1} \ldots s_{k}$, define

$$
a_{w}(\sigma, \nu)=\prod a_{i}\left(\sigma, w_{i} \nu\right) .
$$

Then $a_{w_{0}}(\sigma, \nu)=a(\sigma, \nu)$ from before. Choose a positive definite $W$-invariant inner product on each $V_{\sigma}^{*}$. Because $w_{0}=w_{0}^{-1}$, the operator $a(\sigma, \nu)$ is Hermitian $\left(w_{0} \nu=-\nu\right)$.

Proposition. $\bar{X}(\nu)$ is unitary if and only if a $(\sigma, \nu)$ is positive semidefinite for all $\sigma \in \widehat{W}$.

Proof. This follows from the previous discussion.

Remark. Proposition 2.12 implies that for determining unitarity of any given parameter, it is enough to compute the signatures of the $A(\sigma, \nu)$. Theorem 2.4 is crucial for implementing this calculation by computer; divide the $(-1)$-eigenspace of $w_{0}$ in the dominant chamber into faces according to whether the positive roots are 0 or 1 . Then $\bar{X}(\nu)$ is unitary if and only if it is unitary for any other $\nu$ on that face. Thus, it is sufficient to choose a sample point on each face of the root hyperplane arrangement, and calculate the signature of $a(\sigma, \nu)$ for all $\sigma \in \widehat{W}$.

\section{Type B/C with Unequal Parameters}

3.1. We will consider the particular case when the root system is of type $B_{n}$, and

$$
c_{\alpha}=\left\{\begin{array}{cl}
c & \text { if } \alpha \text { is short } \\
1 & \text { if } \alpha \text { is long. }
\end{array}\right.
$$


We call this algebra $\mathbb{H}_{c}\left(B_{n}\right)$. The general case reduces to (3.1.1) by rescaling the parameters. In particular, $c=1$ is the case of Iwahori-Hecke algebra of type $\mathrm{B}$, while $c=1 / 2$ is identical to the case of the Iwahori-Hecke algebra of type $\mathrm{C}$ with $c_{\alpha}=1$. Aside from the p-adic case, these Hecke algebras are related to the spherical unitary dual of $U(p, q)$ [Ba1].

We emphasize again, that since we only deal with the spherical unitary dual, the explicit determination of the tempered representations as defined in 2.7 is not necessary. For example, the reduction to real infinitesimal character has an elementary proof. Let $\nu=\operatorname{Re} \nu+\sqrt{-1} \operatorname{Im} \nu$ be a parameter such that

$$
\langle\text { Re } \nu, \alpha\rangle \geq 0 \text {. }
$$

Define the standard parabolic subgroup $P=M N$ with Lie algebra $\mathfrak{p}=\mathfrak{m}+\mathfrak{n}$ so that

$$
R_{\mathfrak{n}}=\{\alpha:\langle\operatorname{Re} \nu, \alpha\rangle>0\}, \quad R_{\mathfrak{m}}=\{\alpha:\langle\operatorname{Re} \nu, \alpha\rangle=0\} .
$$

Then the standard module $X(\nu)$ has a unique irreducible quotient $L(\nu)$, and the corresponding objects $X_{M}(\nu)$ and $L_{M}(\nu)$ on $M$ have the same properties. In addition, up to the unitary character $\operatorname{Im} \nu, L_{M}(\nu)$ has real infinitesimal character.

\section{Proposition.}

$$
L(\nu)=\operatorname{Ind}_{P}^{G}\left[L_{M}(\nu)\right] .
$$

In particular, $L(\nu)$ is unitary if and only if $L_{M}(\nu)$ is unitary.

Proof. The Weyl group element satisfying $w \nu=-\bar{\nu}$ fixes $\operatorname{Im} \nu$, so belongs to $W(M)$. Thus the intertwining operator $A_{w}(\nu)$ is induced from the corresponding $A_{M, w}(\nu)$ on $M$. The result follows; we omit further details.

We use the realization of the roots in $\mathfrak{a} \cong \mathbb{R}^{n}\left(\Pi \subset \mathcal{R}^{+} \subset \mathfrak{a}\right)$ given by

$$
\begin{aligned}
\mathcal{R}^{+} & =\left\{-\epsilon_{i}+\epsilon_{j}, \epsilon_{i}+\epsilon_{j}, \epsilon_{i} 1 \leq i<j \leq n\right\}, \\
\Pi & =\left\{\epsilon_{1},-\epsilon_{i}+\epsilon_{i+1}\right\} .
\end{aligned}
$$

A dominant parameter $s$ is then represented by

$$
s=\left(\nu_{1}, \ldots, \nu_{n}\right), \quad 0 \leq \nu_{1} \leq \cdots \leq \nu_{n} .
$$

3.2. We will focus on the case

$$
\langle s, \alpha\rangle \neq c_{\alpha}, \text { for all } \alpha \in \mathcal{R}^{+},
$$

when the standard module $X(s)$ is irreducible. We call it the generic case.

This definition is motivated by the results in [BM3]. For a split p-adic group, the Iwahori-spherical generic representations (in the sense that they admit Whittaker models) are precisely the subquotients of unramified principal series which contain the Steinberg representation of the maximal compact $K$. At the level of Hecke algebras (with $c_{\alpha}=1$, for all $\alpha$ ), the condition is that the corresponding 
module should contain the sign representation of $W$. In particular, it is proven in [BM3] that the generic spherical representations are the irreducible spherical principal series.

Before we give a version of the main result, we recall the notion of irreducible deformation of a parameter. Let $I(\nu)$ be a finite dimensional module so that the action depends analytically on the parameter $\nu$. Assume that $I(\nu)$ is irreducible Hermitian in a path connected region $C \subset \mathbb{R}^{n}$. Then the signature is constant in this region, so determined by its value at a particular $\nu_{0}$. We say that $I(\nu)$ can be deformed irreducibly to $\nu_{0}$, if there is a path connected region $C$ containing $\nu, \nu_{0}$, and satisfying the above conditions. When $C \subset \mathbb{R}$, we say that $\nu$ can be deformed upwards to $\nu_{0}$ if $\nu \leq \nu_{0}$, and downwards if $\nu \geq \nu_{0}$. Finally, if $\nu_{0} \in \bar{C}$,

(1) if $I\left(\nu_{0}\right)$ has a nonunitary factor, then $I(\nu)$ is not unitary,

(2) if $I(\nu)$ is unitary, then every irreducible factor of $I\left(\nu_{0}\right)$ is unitary.

Theorem. A generic spherical representation is unitary if and only if it is a complementary series. In other words, $X(s)$ is unitary if and only if $s$ can be deformed irreducibly to a point such that $X(s)$ is unitarily and irreducibly induced from a unitary generic spherical parameter on a proper Levi component.

A combinatorial description of the unitary parameters can be found in section 3.6. The proof will be given over several sections.

3.3. Recall that representations of $W=W\left(C_{n}\right)=W\left(B_{n}\right)$ are parametrized by pairs of partitions (see [L1])

$$
(a) \times(b)=\left(a_{1}, \ldots, a_{p}\right) \times\left(b_{1}, \ldots, b_{p^{\prime}}\right), \quad \sum a_{i}+\sum b_{j}=n .
$$

The representation $\sigma$ parametrized by 3.3 .1 is obtained as follows. Let $k=$ $\sum a_{i}, l=\sum b_{j}$. Recall that $W \cong S_{n} \ltimes \mathbb{Z}_{2}^{n}$. Let $\xi$ be the character of $\mathbb{Z}_{2}^{n}$ which is trivial on the first $k \mathbb{Z}_{2}$ 's and sign on the remaining $l$. Its centralizer in $S_{n}$ is $S_{k} \times S_{l}$. Let $\sigma_{1}$ and $\sigma_{2}$ be the representations of $S_{k}, S_{l}$ corresponding to the partitions $(a)$ and $(b)$. Then $\sigma$ is $\operatorname{Ind}_{\left(S_{k} \times S_{l}\right) \times \mathbb{Z}_{2}^{n}}^{W}\left[\left(\sigma_{1} \otimes \sigma_{2}\right) \otimes \xi\right]$.

In particular, $(n) \times(0)$ denotes the trivial representation, $(0) \times\left(1^{n}\right)$ the sign representation, and $(n-1) \times(1)$ the reflection representation of $W$ on the Cartan subalgebra.

Recall the setting of section 2.12. The Hermitian matrix associated to a representation $\sigma \in \widehat{W}$, is

$$
a(\sigma, \nu)=\prod\left(\sigma^{*}\left(t_{s_{\alpha_{i}}}\right)\left\langle-w_{i} \nu, \alpha_{i}\right\rangle-c_{\alpha_{i}}\right)\left(\left\langle-w_{i} \nu, \alpha_{i}\right\rangle-c_{\alpha_{i}}\right)^{-1}
$$


The individual terms in the product in (3.3.2) can be rewritten as

$$
a_{i}(\sigma, \nu)= \begin{cases}1 & \text { on the }(+1) \text {-eigenspace of } s_{\alpha_{i}} \text { on } \sigma^{*}, \\ \frac{c_{\alpha_{i}}-\left\langle w_{i} \nu, \alpha_{i}\right\rangle}{c_{\alpha_{i}}+\left\langle w_{i} \nu, \alpha_{i}\right\rangle} & \text { on the }(-1) \text {-eigenspace of } s_{\alpha_{i}} \text { on } \sigma^{*} .\end{cases}
$$

Note that $a(\sigma, \nu) \equiv 1$, for $\sigma=(n) \times(0)$.

An open connected component in the complement of the arrangement of hyperplanes (3.2.1) will be called region. We prove first that the unbounded (open) regions are not unitary.

Lemma. If the open region $\mathcal{F}$ is unbounded, and $\nu \in \mathcal{F}$, then the operator a $(\sigma, \nu)$, for $\sigma=(n-1) \times(1)$, is not positive definite.

Proof. This result holds in general with $\sigma$ the reflection representation. We do not assume that the root system is type $\mathrm{B} / \mathrm{C}$, but we do assume that $w_{0}$ acts by $-I d$ on the Cartan subalgebra for simplicity. For special values of the parameter $c>0$ (in particular $c=\frac{1}{2}, 1$ ) this fact follows from the results in $[\mathrm{KZ}]$ on the signature of Hemitian forms for real Lie groups.

Since the operator $a(\sigma, \nu)$ does not change sign inside the open region $\mathcal{F}$, it is sufficient to prove the statement for one particular $\nu$. The region $\mathcal{F}$ is unbounded, therefore there must exist a simple root $\alpha$ and a point $x \in \mathcal{F}$, such that $\langle\alpha, x\rangle>c_{\alpha}$ and $\left\{x+\underline{\nu} \omega_{\alpha}: \underline{\nu}>0\right\} \subset \mathcal{F}\left(\omega_{\alpha}\right.$ denotes the fundamental coweight corresponding to $\alpha)$. Since we assumed $w_{0}=-I d$,

$$
w_{0} \omega_{\alpha}=-\omega_{\alpha}, \quad w_{0} \alpha=-\alpha, \quad w_{0} x=-x .
$$

We use the canonical realization of the reflection representation $\sigma:=r e f l$ on the Cartan subalgebra, and compute

$$
\left\langle a(\sigma, \nu) \omega_{\alpha}, \omega_{\alpha}\right\rangle_{\sigma} .
$$

We will show that this is negative for large enough $\underline{\nu}$. Let $\mathfrak{p}=\mathfrak{m}+\mathfrak{n}$ be the subalgebra determined by $\omega_{\alpha}$ :

$$
R_{\mathfrak{m}}=\left\{\beta:\left\langle\beta, \omega_{\alpha}\right\rangle=0\right\}, \quad R_{\mathfrak{n}}=\left\{\beta:\left\langle\beta, \omega_{\alpha}\right\rangle>0\right\} .
$$

The long Weyl group element decomposes $w_{0}=w_{m} w^{M}$. Then

$$
a(\sigma, \nu)=a_{w_{m}}\left(\sigma, w^{M} \nu\right) a_{w^{M}}(\sigma, \nu) .
$$

Let $v_{\alpha} \in V_{\sigma}$ be the vector corresponding to $\omega_{\alpha}$. Then since $\left\langle\omega_{\alpha}, \gamma\right\rangle=0$, and therefore $s_{\gamma} \omega_{\alpha}=\omega_{\alpha}$ for all $\gamma \in R_{\mathfrak{m}}$,

$$
a(\sigma, \nu) v_{\alpha}=a_{w_{m}}\left(\sigma, w^{M} \nu\right) v_{\alpha}
$$

In turn this equals

$$
\frac{1}{\prod_{\beta \in R_{\mathfrak{n}}}\left(c_{\beta}+\langle\nu, \beta\rangle\right)} \cdot\left[\underline{\nu}^{\left|R_{\mathfrak{n}}\right|} \prod_{\beta \in R_{\mathfrak{n}}}\left\langle\beta, \omega_{\alpha}\right\rangle t_{w_{m}} v_{\alpha}+\text { lower order terms in } \underline{\nu}\right] .
$$


The factor in front is positive, and so is the coefficient of $t_{w_{m}} v_{\alpha}$, because $\left.\left\langle\beta, \omega_{\alpha}\right\rangle\right\rangle$ 0 , for $\beta \in R_{\mathfrak{n}}$. Since $t_{w_{m}} v_{\alpha}=t_{w_{0}} v_{\alpha}=-v_{\alpha}$, it follows that

$$
\left\langle a(\sigma, \nu) \omega_{\alpha}, \omega_{\alpha}\right\rangle_{\sigma}<0
$$

for large enough $\underline{\nu}$.

Proposition. If $\nu_{n}>\max \{c, 1\}$, then the Hermitian form is indefinite on one of the W-types

$$
(n-1) \times(1), \quad(n-1,1) \times(0) .
$$

Proof. The dimension of the $(+1)$-eigenspace of any $s_{\alpha}$ on $(n-1) \times 1$ is $n-1$, while the dimension of the $(-1)$-eigenspace is 1 . The dimension of the representation $\sigma=(n-1,1) \times(0)$ is $n-1$. The $(+1)$-eigenspaces, $V_{\sigma}^{(1)}$, satisfy

$$
\operatorname{dim}\left(V_{\sigma}^{(1)}\right)=\left\{\begin{array}{l}
n-2 \text { if } \alpha \text { is long, } \\
n-1 \text { if } \alpha \text { is short. }
\end{array}\right.
$$

In particular, we can compute the determinant of $a(\sigma, \nu)$. We get

$$
\begin{array}{lr}
\prod_{i<j} \frac{1-\left(-\nu_{i}+\nu_{j}\right)}{1+\left(-\nu_{i}+\nu_{j}\right)} \frac{1-\left(-\nu_{i}+\nu_{j}\right)}{1+\left(-\nu_{i}+\nu_{j}\right)} \cdot \prod \frac{c-\nu_{i}}{c+\nu_{i}} & \text { for }(n-1) \times(1) \\
\prod_{i<j} \frac{1-\left(-\nu_{i}+\nu_{j}\right)}{1+\left(-\nu_{i}+\nu_{j}\right)} \frac{1-\left(-\nu_{i}+\nu_{j}\right)}{1+\left(-\nu_{i}+\nu_{j}\right)} & \text { for }(n-1,1) \times(0) .
\end{array}
$$

Thus for $X(s)$ to be unitary, there must be an even number of $\nu_{i}>c$. Assume this is the case.

Assume $\nu_{n} \geq \max \{c, 1\}$. Deform $\nu_{n}$ upwards. If there is no $\nu_{i}$ such that $\pm \nu_{i}+\nu_{n}=1$, the region must be unbounded, so by the previous lemma, the module cannot be unitary, and the form is indefinite on $(n-1) \times(1)$. Thus deform $\nu_{n}$ upwards until the first time it is equal to $\nu_{i}+1$ for some $i$. Consider the Levi component $M$ of type $A_{1}$ given by the root $-\epsilon_{i}+\epsilon_{n}$. We can conjugate $s$ so that $\nu_{i}, \nu_{n}$ are on the coordinates $n-1$ and $n$ respectively. This $s$ determines a character $\chi$ on $M$ so that $\bar{X}(s)$ is the spherical subquotient of the induced module

$$
\operatorname{Ind}_{M}^{G}(\chi)=\mathbb{H} \otimes_{\mathbb{H}_{M}} \chi
$$

The character $\chi$ of $\mathbb{H}_{M}$ is $\chi=\operatorname{triv} \otimes \mathbb{C}_{\nu}$. The module $\operatorname{Ind} d_{M}^{G}(\chi)$ is in fact irreducible (so equal to $\bar{X}(s)$ ). Its Hermitian dual is $\operatorname{Ind}_{M}^{G}\left(\chi^{-1}\right)$, so the invariant Hermitian form is given by an intertwining operator of the type defined in section 2.11, despite the fact that the trivial representation is not tempered. The shortest element $w \in W$ that takes $\nu$ to $-\nu$, and fixes the trivial representation of $M$, can be decomposed into a product of factors similar to the decomposition (2.11.3). 
There are several types of $A_{\alpha}$ as in (2.11.1). One type is just an $a_{i}(\sigma, \nu)$ as in (2.12.6). The second type is induced from an operator on an $M_{\alpha}$ of type $A_{2}$, with $M$ of type $A_{1}$, and a third one is induced from an $M_{\alpha}$ of type $B_{2}$, with $M$ of type $A_{1}($ long $)$. We denote the last two as

$$
A_{1} \subset A_{2}, \quad A_{1}(\text { long }) \subset B_{2} .
$$

Example. Consider the Hecke algebra of rank 3. The simple roots are $\left\{\epsilon_{1},-\epsilon_{1}+\right.$ $\left.\epsilon_{2},-\epsilon_{2}+\epsilon_{3}\right\}$. Let $M=A_{1}$ be the Levi component corresponding to the root $\alpha_{2}=-\epsilon_{1}+\epsilon_{2}$ and let $\sigma$ be the trivial representation. The parameter is $s=$ $(-1 / 2,1 / 2,0)+\nu$, where $\nu=\left(\nu_{1}, \nu_{1}, \nu_{2}\right)$, with $0 \leq \nu_{1} \leq \nu_{2}$. The shortest element that takes $\nu$ to $-\nu$, and fixes the trivial representation of $\mathbb{H}_{M}$ is $w=s_{2} w_{0}$. The intertwining operator

$$
A_{m}(M, \sigma, \nu): \mathbb{H} \otimes_{\mathbb{H}_{M}} \sigma \mathbb{1}_{\nu} \rightarrow \mathbb{H} \otimes_{\mathbb{H}_{M}} \sigma \mathbb{1}_{-\nu}
$$

is given by multiplication with $r_{w}$. The Weyl group element $w$ decomposes into a product $w_{\alpha_{2}} \cdot s_{1} \cdot w_{\alpha_{3}} \cdot w_{\alpha_{1}}$. In terms of simple reflections $w_{\alpha_{1}}=s_{1} s_{2} s_{1}$, $w_{\alpha_{3}}=s_{3} s_{2}$ and $w_{\alpha_{2}}=s_{2} s_{3}$. The intertwining operator decomposes accordingly into a product

$$
\begin{aligned}
& A_{\alpha_{2}}\left(-\nu_{2},-1 / 2-\nu_{1}, 1 / 2-\nu_{1}\right) \circ a_{\alpha_{1}}\left(\nu_{2},-1 / 2-\nu_{1}, 1 / 2-\nu_{1}\right) \\
& \circ A_{\alpha_{3}}\left(-1 / 2-\nu_{1}, 1 / 2-\nu_{1}, \nu_{2}\right) \circ A_{\alpha_{1}}\left(-1 / 2+\nu_{1}, 1 / 2+\nu_{1}, \nu_{2}\right) .
\end{aligned}
$$

The factors $A_{\alpha_{2}}$ and $A_{\alpha_{3}}$ are induced from $A_{1} \subset A_{2}$, the factor $A_{\alpha_{1}}$ is induced from $A_{1}$ (long) $\subset B_{2}$, while the factor $a_{\alpha_{1}}$ corresponds to a single $r_{\alpha_{1}}$.

Returning to the general case, we can compute the determinant of the form. For $(n-1) \times(1)$ we get a product of factors

$$
\begin{aligned}
& \frac{1-\left( \pm \nu_{k}+\nu_{l}\right)}{1+\left( \pm \nu_{k}+\nu_{l}\right)} \quad k, l \neq i, n, \\
& \frac{c-\nu_{k}}{c+\nu_{k}} \quad k \neq i, n, \\
& \frac{c-\nu_{i}}{c+\nu_{n}} \quad \text { for } A_{1}(\text { long }) \subset B_{2}, \\
& \frac{1-\left(\nu_{k}-\nu_{n}\right)}{1-\left(-\nu_{k}+\nu_{i}\right)} \quad \text { for } A_{1} \subset A_{2} .
\end{aligned}
$$


These formulas come from direct calculations for the Hecke algebras of rank 2 . See [Ba2] for some more general calculations of this kind. The factor for $A_{1} \subset B_{2}$ comes from the fact that the restriction of $(n-1) \times(1)$ consists of $(1) \times(1)$ and $(2) \times(0)$.

For $(n-1,1) \times(0)$ we get the same factors, except the one for $A_{1} \subset B_{2}$ is missing because the restriction of this representation to $B_{2}$ consists of $(2) \times(0)$ and $(11) \times(0)$, and only the first one occurs in an induced from the trivial character on an $A_{1}$ (long) $\subset B_{2}$.

Comparing these four determinants, we find that the form has to be indefinite if we assume $c-\nu_{n}<0$.

3.4. We prove theorem 3.2 in the case when $0<c \leq 1$. By proposition 3.3, a necessary condition for unitarity is that $\nu_{n}<1$. Assume that $c \leq \nu_{n}<1$. Proceeding as in the proof of proposition 3.3, deform $\nu_{n}$ upwards until the first reducibility hyperplane. If this is of the form $\nu_{n}-\nu_{i}=1$, the argument in 3.3 applies to show that the form is indefinite.

Assume the first reducibility hyperplane is $\nu_{n}+\nu_{i}=1$. The only difference between this case and the proof of proposition is that in order for the parameter $s$ to be in dominant form for the Levi component $M$ of type $A_{1}$ (given by the root $\epsilon_{i}+\epsilon_{n}$ ), we need to conjugate $\nu_{i}$ to $-\nu_{i}$. This has the effect that the formula for the factor $A_{1}($ long $) \subset B_{2}$ in (3.3.9) becomes $\frac{c+\nu_{i}}{c+\nu_{n}}$. Comparing as before the four determinants on $(n-1) \times(1)$ and $(1, n-1) \times(0)$, it follows that

$$
\frac{c-\nu_{i}}{c-\nu_{n}}>0 \text {. }
$$

Since we assumed that $\nu_{n}>c$, it implies that also $\nu_{i}>c$. The reducibility hyperplane being $\nu_{n}+\nu_{i}=1$, necessarily then $c<1 / 2$.

We summarize this discussion in the following proposition.

Proposition. If $1 / 2<c \leq 1$, and $\nu_{n}>c$, then the Hermitian form is indefinite on one of the $W$-types $(n-1) \times(1)$ or $(n-1,1) \times(0)$.

If $0<c \leq 1 / 2$, and $\nu_{n}>c$, a necessary condition for unitarity is that there exists $i<n$, such that $1-\nu_{i+1}<\nu_{n}<1-\nu_{i}$ and $\nu_{i}>c$.

When $\nu_{n} \leq 1 / 2$, any $\nu_{i}$ can be deformed to $\nu_{i+1}$ without any reducibility occuring. Then the module is induced irreducible from a Hermitian module on a Levi component $M$ of type $g l(2) \times \mathfrak{g}(n-2)$, where $\mathfrak{g}(n-2)$ is the Lie algebra of type $B$ of rank $n-2$. The module on $g l(2)$ is a unitary complementary series for $g l(2)$ with parameter $\left(\nu_{i+1}, \nu_{i+1}\right)$, while the parameter on $\mathfrak{g}(n-2)$ is obtained from $s$ by removing the two coordinates $\nu_{i}, \nu_{i+1}$. Similarly it is possible to deform $\nu_{1}$ to 0 without any reducibility; the resulting module is unitarily induced from a parameter on a Levi component of the form $g l(1) \times \mathfrak{g}(n-1)$. The parameter 
on $g l(1)$ is 0 , and on $\mathfrak{g}(n-1)$ it is obtained from $s$ by removing $\nu_{1}$. Proceeding by induction on rank, the result follows.

Now consider the case $1 / 2<c \leq 1$. By proposition 3.4, we may as well assume that

$$
0<\nu_{1}<\cdots<\nu_{n}<c .
$$

By the earlier argument, there must be a $k$ such that

$$
0<\nu_{1}<\cdots<\nu_{k} \leq 1 / 2<\nu_{k+1}<\cdots<\nu_{n}<c \leq 1 .
$$

Otherwise, the earlier argument reduces considerations to a smaller rank algebra. Suppose $s$ cannot be deformed to a unitarily induced parameter from a smaller group. Then there must be $k_{1}>k$ such that

$$
0<1-\nu_{k_{1}}<\nu_{1}
$$

and for each $1 \leq i \leq k$, there must be a $k<k_{i}$ such that

$$
\nu_{i}<1-\nu_{k_{i}}<\nu_{i+1} \text {. }
$$

Thus $k \leq n-k$. On the other hand, if for any $i \geq j$, we can deform $\nu_{i}$ to $\nu_{i+1}$ without going through any reducibility point, the resulting module is unitarily induced irreducible from a Hermitian module on a $g l(2) \times \mathfrak{g}(n-2)$, such that the parameter on $g l(2)$ is not unitary. Thus for any $j \geq k$ there must be $k_{j}<k$ such that

$$
\nu_{j}<1-\nu_{k_{j}}<\nu_{j+1}
$$

It follows that $k>n-k$.

It remains to analyze the case $0<c<1 / 2$. If $\nu_{n}<c<1 / 2$, the previous argument for $\nu_{n} \leq 1 / 2$ applies. Assume $\nu_{n}>c$. By proposition 3.4 above, the region must be of the form

$$
\begin{aligned}
& 0 \leq \nu_{1} \leq \cdots \leq \nu_{j}<c<\nu_{j+1} \leq \cdots \leq \nu_{n}<1-\nu_{i}, \text { or } \\
& c<\nu_{1} \leq \cdots \leq \nu_{n}<1-\nu_{i},
\end{aligned}
$$

where the essential remark is that, in the first type of regions, $i>j$ (because $\left.\nu_{i}>c\right)$.

We claim that a parameter in any such region can be deformed irreducibly to a unitarily induced parameter from a smaller algebra. In the first case, since $\nu_{1}+\nu_{k}<\nu_{i}+\nu_{n}<1$, one can deform $\nu_{1}$ irreducibly to 0 .

In the second case, the same argument as in (3.4.3)-(3.4.6) works to show that there exists $l$ such that one can deform $\nu_{l}$ irreducibly to $\nu_{l+1}$.

Note also that if there is an odd number of $\nu_{l}>c$, the region cannot be unitary. By the deformation argument, if this were the case, one would reduce it to a region in $B_{1}$ with $\nu>c$, which is not unitary. 
In summary, we have proved a generalization of theorem 3.2 in [Ba2]. Write the general parameter as before $0 \leq \nu_{1} \leq \nu_{2} \leq \cdots \leq \nu_{n}$.

Theorem. The complementary series for type $B_{n}$ with parameter $0<c \leq 1$ is $1 / 2<\mathrm{c} \leq 1$ :

$$
0 \leq \nu_{1} \leq \cdots \leq \nu_{k} \leq 1 / 2<\nu_{k+1}<\nu_{k+2}<\cdots<\nu_{n}<c
$$

so that $\nu_{i}+\nu_{j} \neq 1$ for $i \neq j$ and there are an even number of $\nu_{i}$ such that $1-\nu_{k+1}<\nu_{i}<c$ and an odd number of $\nu_{i}$ such that $1-\nu_{k+j+1}<\nu_{i}<1-\nu_{k+j}$. $\mathbf{0}<\mathbf{c} \leq \mathbf{1} / \mathbf{2}$ :

$$
0 \leq \nu_{1} \leq \cdots \leq \nu_{j}<c<\nu_{j+1} \leq \cdots \leq \nu_{n}<1-\nu_{j+1},
$$

where $j$ could be 0 (i.e., all entries could be $>c$ ) and

(1) $n-j$ is even (there is an even number of entries $>c$ );

(2) $\nu_{j+1} \leq \cdots \leq \nu_{k} \leq 1 / 2<\nu_{k+1}<\cdots<\nu_{n}<1$ satisfy $\nu_{l}+\nu_{l^{\prime}} \neq 1$ for $j<$ $l \neq l^{\prime} \leq n$ and there are an even number of $\nu_{i}$ such that $1-\nu_{k+1}<\nu_{i}<1$ and an odd number of $\nu_{i}$ such that $1-\nu_{k+l+1}<\nu_{i}<1-\nu_{k+l}$. .

Example. In the Hecke algebra of type $B_{2}$, with $0<c \leq 1$, the unitary generic spherical parameters are:

$$
\begin{array}{ll}
0<c<1 / 2: & 0 \leq \nu_{1} \leq \nu_{2}<c \quad \text { and } \quad c<\nu_{1} \leq \nu_{2}<1-\nu_{1} . \\
1 / 2 \leq c \leq 1: & 0 \leq \nu_{1}<\nu_{2}<\min \left\{c, 1-\nu_{1}\right\} .
\end{array}
$$

3.5. We now consider the case $c>1$. Recall $s=\left(\nu_{1}, \ldots, \nu_{n}\right)$, and because of section 3.3 we assume that

$$
0<\nu_{1}<\cdots<\nu_{n}<c .
$$

Because of the arguments in section 3.4, we may as well assume $1 \leq \nu_{n}$.

Let $x$ be the half-integer satisfying $\nu_{n}-1 / 2<x \leq \nu_{n}$. Let

$$
s^{\prime}:=\left(\nu_{1}, \ldots, \nu_{n-1}\right) .
$$

Let $\chi(x, t)$ be the character on $g l(2 x)$ corresponding to the coordinates

$$
\chi(x, t):=(x-1 / 2-t, \ldots,-x+1 / 2-t)
$$

Denote the induced module

$$
X(s, x, t):=\operatorname{Ind}_{B(n) \times g l(2 x)}^{B(n+2 x)}[s \otimes \chi(x, t)] .
$$

\section{Proposition.}

(1) The module $X(s, x, t)$ is irreducible for $0 \leq t \leq x-\nu_{n}+1 / 2$.

(2) The module $X\left(s^{\prime}, x+1 / 2, u\right)$ is irreducible for $0<u<\nu_{n}-x$. 
Proof. We prove (1), since (2) is essentially the same statement. We also assume that $x$ is an integer for simplicity. We will freely use the irreducibility results about induced modules in type A, as in [Ba2] lemma 3.1. First we show that

$$
\operatorname{Ind}_{g l(x)}^{\mathfrak{g}(x)}[\chi(x / 2, x / 2 \pm t)] \quad \text { is irreducible. }
$$

We consider the case

$$
\chi(x / 2, x / 2+t)=(-1 / 2-t,-3 / 2-t, \ldots,-x+3 / 2-t,-x+1 / 2-t) .
$$

The intertwining operator

$$
\begin{aligned}
& X(1 / 2+t, \ldots, x-3 / 2+t, x-1 / 2+t) \longrightarrow \\
& \quad X(-1 / 2-t, \ldots,-x+3 / 2-t, x-1 / 2+t)
\end{aligned}
$$

is onto the induced module

$$
\operatorname{Ind} d_{g l(x-1) \times g l(1)}^{\mathfrak{g}(x)}[\chi(x / 2-1 / 2,-x / 2+1 / 2-t) \otimes \chi(1 / 2, x-1 / 2+t)]
$$

by induction. Since the intertwining operator

$$
\begin{array}{r}
X_{g l(x)}(-1 / 2-t, \ldots,-x+3 / 2-t, x-1 / 2+t) \longrightarrow \\
\quad X_{g l(x)}(x-1 / 2+t,-1 / 2-t, \ldots,-x+3 / 2-t)
\end{array}
$$

is an isomorphism onto

$$
\operatorname{Ind}_{g l(1) \times g l(x-1)}^{\mathfrak{g}(x)}[\chi(1 / 2, x-1 / 2+t) \otimes \chi(x / 2-1 / 2,-x / 2+1 / 2-t)]
$$

when restricted to the left hand side of (3.5.8), the latter module has a unique irreducible quotient. Because $x-1 / 2+t<c$, the intertwining operator

$$
\begin{gathered}
X(x-1 / 2+t,-1 / 2-t, \ldots,-x+3 / 2-t) \longrightarrow \\
X(-x+1 / 2-t,-1 / 2-t, \ldots,-x+3 / 2-t)
\end{gathered}
$$

maps (3.5.9) onto

$$
\operatorname{Ind} d_{g l(1) \times g l(x-1)}^{\mathfrak{g}(x)}[\chi(1 / 2,-x+1 / 2-t) \otimes \chi(x / 2-1 / 2,-x / 2+1 / 2-t)] .
$$

Finally the intertwining operator

$$
\begin{gathered}
X(-x+1 / 2-t,-1 / 2-t, \ldots,-x+3 / 2-t) \longrightarrow \\
X(-1 / 2-t, \ldots,-x+3 / 2-t,-x+1 / 2-t)
\end{gathered}
$$

maps (3.5.11) onto $\operatorname{Ind}_{g l(x)}^{\mathfrak{g}(x)}[\chi(x / 2,-x / 2-t)]$. So this module has a unique irreducible quotient. But by virtue of being embedded in $X(-1 / 2-t, \ldots,-x+3 / 2-$ $t,-x+1 / 2-t)$, it also has a unique irreducible submodule, so must be irreducible itself. This immediately implies that (3.5.5) is irreducible as well. The proof for $\chi(x / 2, x / 2-t)$ is identical.

Next we show that

$$
\operatorname{Ind} d_{g l(2 x)}^{\mathfrak{g}(2 x)}[\chi(x, t)] \quad \text { is irreducible. }
$$


The intertwining operator

$$
\begin{aligned}
& X(1 / 2-t, 1 / 2+t, \ldots, x-1 / 2-t, x-1 / 2+t) \longrightarrow \\
& \quad X(x-1 / 2-t, \ldots, 1 / 2-t, x-1 / 2+t, \ldots, 1 / 2+t)
\end{aligned}
$$

has image

$$
\operatorname{Ind} d_{g l(x) \times g l(x)}^{\mathfrak{g}(2 x)}[\chi(x / 2, x / 2+t) \otimes \chi(x / 2, x / 2-t)] .
$$

The intertwining operator

$$
\begin{aligned}
& X(x-1 / 2+t, \ldots, 1 / 2+t, x-1 / 2-t, \ldots, 1 / 2-t) \longrightarrow \\
& \quad X(-1 / 2-t, \ldots,-x+1 / 2-t, x-1 / 2-t, \ldots, 1 / 2-t)
\end{aligned}
$$

maps (3.5.15) onto

$$
\operatorname{Ind}_{g l(x) \times g l(x)}^{\mathfrak{g}(2 x)}[\chi(x / 2,-x / 2-t) \otimes \chi(x / 2, x / 2-t)] .
$$

Then the intertwining operator

$$
\begin{aligned}
& X(-1 / 2-t, \ldots,-x+1 / 2-t, x-1 / 2-t, \ldots, 1 / 2-t) \longrightarrow \\
& \quad X(x-1 / 2-t, \ldots,-x+1 / 2-t)
\end{aligned}
$$

maps (3.5.17) onto (3.5.13). Thus (3.5.13) has a unique irreducible quotient. The fact that it also has a unique irreducible submodule follows in the same way as the earlier argument. Embed it in

$$
\operatorname{Ind} d_{g l(x) \times g l(x)}^{\mathfrak{g}(2 x)}[\chi(x / 2, x / 2-t) \otimes \chi(x / 2,-x / 2-t)] .
$$

Changing $\chi(x / 2, x / 2-t)$ to $\chi(x / 2,-x / 2+t)$ is injective, because of (3.5.5). Reordering the coordinates in decreasing order is also injective because of the irreducibility results for $g l$. The claim follows.

To prove the general case, we use the same technique. Let $\nu$ be the parameter of $X(s, x, t)$ made dominant. Then the intertwining operator

$$
X(\nu) \longrightarrow X(x-1 / 2-t, \ldots,-x+1 / 2-t, s)
$$

has image

$$
I n d_{g l(2 x) \times g l(n)}^{\mathfrak{g}(2 x+n)}\left[\chi(x, t) \otimes \bar{X}_{g l(n)}(s)\right] .
$$

The assumptions on $x$ and $t$ imply that this module is isomorphic to

$$
\operatorname{Ind} d_{g l(n) \times g l(2 x)}^{\mathfrak{g}(2 x+n)}\left[\bar{X}_{g l(n)}(s) \otimes \chi(x, t)\right] .
$$

This has $X(s, x, t)$ as a quotient. Thus (3.5.4) has a unique irreducible quotient. To show that it also has a unique irreducible submodule, embed it in (3.5.21), use the irreducibility of $\operatorname{In} d_{g l(2 x)}^{\mathfrak{g}(2 x)}[\chi(x, t)]$ to show that it embeds in $X(-\nu)$.

Corollary. The parameter $s$ is unitary only if $s^{\prime}$ is unitary. 
Proof. Assume $\bar{X}(s)$ is unitary. Then $X(s, x, t)$ is unitary irreducible for $0 \leq$ $t \leq x-\nu_{n}+1 / 2$. At $x-\nu_{n}+1 / 2=t$, the corresponding $\bar{X}(s, x, t)$ is also irreducible unitary, and equal to $X\left(s^{\prime}, x+1 / 2, u\right)$, with $u=\nu_{n}-x$. Then $X\left(s^{\prime}, x+\right.$ $1 / 2,0)$ is unitarily induced irreducible from $\bar{X}(s) \otimes \operatorname{triv}$. Thus $\bar{X}\left(s^{\prime}\right)$ is unitary, as claimed.

3.6. The explicit, combinatorial, description of the unitary generic spherical parameters is given in the following theorem.

Theorem. The complementary series for type $B_{n}$ with parameter $c>1$ is

$$
0 \leq \nu_{1} \leq \nu_{2} \leq \cdots \leq \nu_{m}<1+\nu_{1}<\nu_{m+1}<\nu_{m+2}<\cdots<\nu_{n}<c
$$

satifying the conditions:

(1) $0 \leq \nu_{1} \leq \nu_{2} \leq \cdots \leq \nu_{m}<c$ satisfy the unitarity conditions in Theorem 3.4 for $B_{m}$

(2) $\nu_{j+1}-\nu_{j}>1$ for all $j \geq m+1$;

(3) either $\nu_{m+1}-\nu_{m}>1$ or, if $1-\nu_{k+1}<\nu_{m}<1-\nu_{k}$ (from Theorem 3.4 $k+m$ is necessarily odd), then $1+\nu_{l}<\nu_{m+1}<1+\nu_{l+1}$, with $k \geq l+1$ and $m+l$ even.

In view of Corollary 3.5, the proof is by induction on $n$, and will be given in sections 3.7 and 3.8 .

3.7. There are some regions which need to be discussed first. In each $B_{2 k}$ there is a region $\left(\nu_{1}, \nu_{2}, \ldots, \nu_{2 k}\right)$ given by the inequalities:

$$
\begin{aligned}
& 0 \leq\left|\nu_{2 k-1}-1\right|<\nu_{1}<1-\nu_{2 k-2}<\nu_{2}<\cdots<\nu_{k-2}<1-\nu_{k+1}<\nu_{k-1}< \\
& <\nu_{2 k}-1<\nu_{k}<1-\nu_{k-1}<\nu_{k+1}<1-\nu_{k-2}<\nu_{k+2}<\cdots<\nu_{2 k-2}< \\
& <1-\nu_{1}<\nu_{2 k-1}<1+\nu_{1}<\cdots<1+\nu_{k-1}<\nu_{2 k}<1+\nu_{k} .
\end{aligned}
$$

For example, the region in $B_{4}$ is

$$
0 \leq\left|\nu_{3}-1\right|<\nu_{1}<\nu_{4}-1<\nu_{2}<1-\nu_{1}<\nu_{3}<1+\nu_{1}<\nu_{4}<1+\nu_{2} .
$$

Note that a parameter in a region (3.7.1) with $k>1$ cannot be deformed irreducibly to a unitarily induced irreducible parameter.

Proposition. In $B_{n}, n=2 k$, the form on $(n-2) \times(2),(n-1) \times(1)$, or $(n) \times(0)$ is indefinite in the region (3.7.1). 
Proof. The proof is by induction on $k$. We do the induction step first. Deform the pairs $\left(\nu_{k-2}, \nu_{k-1}\right)$ and $\left(\nu_{k+1}, \nu_{k+2}\right)$ irreducibly to

$$
0<\nu=1-\nu_{k+1}=\nu_{k-1}=\nu_{k-2}=1-\nu_{k+2}<1 / 2 .
$$

If the generic parameter is unitary, then this spherical parameter is unitary as well, because this is the first place where the standard module has become reducible. The spherical representation is induced irreducible from a parameter on $B_{2 k-4} \times g l(4)$. The parameter on $B_{2 k-4}$ is obtained from the original one by removing the above four entries. The parameter on $g l(4)$ is unitary. Thus this parameter is unitary if and only if the parameter on the $B_{2 k-4}$ is unitary. By induction, the parameter is not unitary, so the generic one isn't either. The assertion about the form being indefinite on the specified K-types comes from the fact that the restriction of a W-type $(n-a) \times(a)$ is a sum of W-types $(n-4-b) \times(b)$ with $b \leq a$.

The initial step is formed of the cases $k=1$ and $k=2$. For $k=1$, the parameter is

$$
0<\left|\nu_{2}-1\right|<\nu_{1}<\nu_{2}<1+\nu_{1} .
$$

Deform $\nu_{1}$ upwards to $\nu_{2}$. No reducibility occurs until the endpoint. The resulting parameter is unitarily induced irreducible from a parameter on $g l(2)$, of the form $(-\nu, \nu)$ with $\nu>1 / 2$, because $\nu>|\nu-1|$. This not unitary, and the form is indefinite on the sum of W-types (2) and (11). The claim follows.

For $k=2$, the parameter is (3.7.2). Deform $\nu_{1}$ to $\nu_{2}$, and $\nu_{3}$ to $\nu_{4}$ so that no reducibility occurs in between. If the generic parameter is unitary, then so is the spherical one at the endpoint. The parameter can be written as

$$
(\nu, \nu, \nu+1, \nu+1) \quad \text { with } 0<\nu<1 / 2 .
$$

This parameter is unitarily induced irreducible from $g l(4)$. On $g l(4)$, the parameter is induced from a character on $g l(2) \times g l(2)$, but outside the complementary series. The form is indefinite on the W-types (31) and (22). The claim about the $\mathrm{W}$-types of the generic representation follows from this fact.

3.8. Proof of theorem 3.6. If $\nu_{n}<1+\nu_{1}$, one can deform $\nu_{n}$ irreducibly until $\nu_{n}<1$. Then the same arguments from section 3.4 apply.

Assume therefore that $\nu_{n}>1+\nu_{1}$. The parameter is

$$
0 \leq \nu_{1} \leq \nu_{2} \leq \cdots \leq \nu_{m}<1+\nu_{1}<\nu_{m+1}<\cdots<\nu_{n-1}<\nu_{n}<c .
$$

Assume it is unitary. By corollary 3.5 , the string $\left(\nu_{1}, \nu_{2}, \ldots, \nu_{m}, \ldots, \nu_{n-1}\right)$ satisfies the conditions for unitarity from theorem 3.6. So the parameter for $B_{n}$ satisfies conditions (1). We need to check that conditions (2) and (3) are also satisfied.

There are two cases, $m+1<n$, and $m+1=n$. 
Case 1: $m+1<n$, which also means that $\nu_{n-1}>1+\nu_{1}$. In this case conditon (3) is satisfied by the induction hypothesis, we only need to check (2) for $j=n-1$, same as $\nu_{n}>1+\nu_{n-1}$. If $\nu_{1}$ can be deformed irreducibly to 0 , the resulting parameter is unitarily induced irreducible from the parameter on $B_{n-1}$ obtained by removing $\nu_{1}$. The induction hypothesis applied to $\left(\nu_{2}, \ldots, \nu_{n}\right)$ implies the claim for $B_{n}$.

If $\nu_{1}$ cannot be deformed to 0 irreducibly, then $\nu_{n-1}>1+\nu_{n-2}$. Necessarily $\nu_{n}>1+\nu_{n-1}$, so the parameter on $B_{n}$ satisfies condition (2). This is because otherwise one could deform $\nu_{n-1}$ to $\nu_{n}$ irreducibly, and the resulting parameter would be unitarily induced from $B_{n-2} \times g l(2)$, with the parameter on $g l(2)$ nonunitary.

Case 2: $m+1=n$, which also means $\nu_{n-1}<1+\nu_{1}$. In this case we only need to check that (3) is satisfied. The parameter is

$$
0 \leq \nu_{1} \leq \cdots \leq \nu_{n-1}<1+\nu_{1}<\nu_{n}<c .
$$

If we can deform $\nu_{1}$ to 0 , the proof follows by unitary induction from $B_{n-1}$. Otherwise, necessarily $0<\left|1-\nu_{n-1}\right|<\nu_{1}$. From the conditions of unitarity of theorems 3.6 and 3.4 for $0<\nu_{1}<\cdots<\nu_{n-1}$, there exists $k, 1 \leq k \leq n-2$, such that $\nu_{k}<1-\nu_{k-1}<\nu_{k+1}$. We can write the inequalities as

$$
\begin{aligned}
& 0<\left|1-\nu_{n-1}\right|<\nu_{1} \leq \nu_{2} \leq \cdots \leq \nu_{k-1} \leq \nu_{k}<1-\nu_{k-1}<\nu_{k+1}<\ldots \\
& \cdots<\nu_{n-2}<1-\nu_{1}<\nu_{n-1}<1+\nu_{1}<\cdots<1+\nu_{l}<\nu_{n}<1+\nu_{l+1},
\end{aligned}
$$

for some $l$. If we can deform any $\nu_{j+1}$ down to $\nu_{j}$ with $j \leq k-1$ irreducibly, the resulting parameter would be unitarily induced irreducible from $B_{n-2} \times g l(2)$ with a unitary $g l(2)$ parameter. The induction hypothesis for the parameter on $B_{n-2}$ with $\nu_{j}, \nu_{j+1}$ removed, implies that condition (3) holds for $B_{n}$. So assume this is not possible. Then $l=k-1$, otherwise we could deform $\nu_{k}$ down to $\nu_{k-1}$. We are reduced to the case when the inequalities are

$$
\begin{gathered}
0<\left|1-\nu_{n-1}\right|<\nu_{1} \leq \nu_{2} \leq \cdots \leq \nu_{k-1}<\nu_{n}-1<\nu_{k}<1-\nu_{k-1}<\nu_{k+1}< \\
<\nu_{n-2}<1-\nu_{1}<\nu_{n-1}<1+\nu_{1}<\cdots<1+\nu_{k-1}<\nu_{n}<1+\nu_{k} .
\end{gathered}
$$

For each pair $\nu_{j}, \nu_{j+1}$, with $1 \leq j \leq k-2$ and $k+1 \leq j \leq n-3$, there is $\ell_{j}$ such that $\nu_{j}<1-\nu_{\ell_{j}}<\nu_{j+1}$. For $\nu_{1}<\nu_{2}<\cdots<\nu_{k-1}$, there are $k-2$ inequalities and the possible $\ell_{j}$ are $k+1, \ldots, n-2$. This means $n-2-k \geq k-2$ or, equivalently $n \geq 2 k$. For $\nu_{k+1}<\cdots<\nu_{n-2}$, there are $n-k-3$ inequalities and the possible $\ell_{j}$ are $\ell_{j}=2, \ldots, k-2$. Then $k-3 \geq n-k-3$, and so $n \leq 2 k$.

We conclude that $n=2 k$, and the parameter is as in (3.7.1). The nonunitarity of such parameters was discussed in section 3.7. Thus to be unitary, the parameter has to satisfy the condition that it can be deformed irreducibly to one which is unitarily induced from a proper Levi component, which is the content of the conditions in theorem 3.6. 


\section{The Unitary Groups}

4.1. One of the results in [Ba1], is that the spherical unitary dual of the unitary quasisplit groups $U(n, n)$ and $U(n+1, n)$ matches the spherical unitary dual of the Hecke algebras of type $B_{n}$ with parameters $c=1 / 2$ and $c=1$ respectively. The results in this paper imply similar results for $U(p, q)$ with $|p-q|>1$. Let $c=\frac{p-q+1}{2}$. The spherical parameters for $U(p, q)$ are matched with the spherical parameters of $\mathbb{H}_{c}\left(B_{n}\right)$ with $n=q$, the real rank of $U(p, q)$. A spherical representation of $U(p, q)$ is called generic, if the standard module is irreducible (in the quasisplit cases, these are the spherical representations with Whittaker models, $c f$. [V2]). In coordinates,

$$
\nu_{j} \pm \nu_{i} \notin \mathbb{Z}_{>0}, \nu_{i}-c \notin \mathbb{Z}_{\geq 0}, \quad 1 \leq i, j \leq n .
$$

Theorem. A generic parameter for $U(p, q)$ is unitary only if it is unitary for $\mathbb{H}_{c}\left(B_{n}\right)$.

Proof. Let $\nu$ be a spherical parameter, and denote by $M$ the Levi component of the minimal real parabolic subgroup of $U(p, q)$. As explained in [Ba1], the classification of spherical irreducible and Hermitian $(\mathfrak{g}, K)$ modules for $U(p, q)$ coincides with that for $\mathbb{H}_{c}\left(B_{n}\right)$. In particular, if $(\mu, V)$ is a $K$-type, then there is a Hermitian operator

$$
a(\mu, \nu):\left(V^{M}\right)^{*} \longrightarrow\left(V^{M}\right)^{*}
$$

with the property that a parameter is unitary if and only if $a(\mu, \nu)$ is positive semidefinite. The module $\left(V^{M}\right)^{*}$ is endowed with a representation of $W$, call it $\sigma(\mu)$. In section 4.9 of [Ba1], a set of K-types called relevant is defined. A relevant $\mathrm{K}$-type has the property that the Hermitian matrix in (4.1.2) coincides with $a(\sigma(\mu), \nu)$ in (3.3.2). Furthermore the $\sigma(\mu)$ occuring contain the list used in theorem 3.6 to establish which generic parameters are unitary. Thus the generic parameters for $U(p, q)$ that are unitary, have to be contained in the set of parameters which are unitary for $\mathbb{H}_{c}\left(B_{n}\right)$.

For $|p-q|>2$, the spherical generic unitary duals of $U(p, q)$ and $\mathbb{H}_{c}\left(B_{n}\right)$ do not coincide, as seen in the next example. It is likely that for $|p-q|=2$, the unitary duals coincide, but we have not checked the details.

Example. In the case of $U(p, 2)$, the spherical unitary dual is well-known. The following explicit description can be found for example in [KS].

Theorem $([\mathrm{KS}])$. The spherical unitary parameters $\left(\nu_{1}, \nu_{2}\right), 0 \leq \nu_{1} \leq \nu_{2}$ for $U(p, 2)$ are:

(1) the triangles $\nu_{2}-\nu_{1} \geq k, \nu_{2}+\nu_{1} \leq k+1$ for $k \geq 0$ with $\nu_{2} \leq \frac{p-1}{2}$; 
(2) the lines $\nu_{2}-\nu_{1}=k$ for $k \geq 1$ with $\nu_{2} \leq \frac{p-1}{2}$.

(3) the point $\left(\nu_{2}, \nu_{1}\right)=\left(\frac{p+1}{2}, \frac{p-1}{2}\right)$ (the trivial representation).

On the other hand, the generic spherical unitary dual for the Hecke algebra $\mathbb{H}_{c}\left(B_{2}\right)$ with $c=\frac{p-1}{2}$ is formed by two triangles $\nu_{2}+\nu_{1}<1$ and $\nu_{2}-\nu_{1}>1, \nu_{2}<c$.

The two pictures below illustrate the case $p=8$. The figures show the triangle $0 \leq \nu_{1} \leq \nu_{2} \leq \frac{p-1}{2}=\frac{7}{2}$ (all generic spherical unitary parameters are inside this triangle) and all the lines of reducibility inside it. The open regions are green if they parametrize unitary representations and white otherwise. On the lines, green means that the spherical representation is unitary and red indicates that is not. Outside this triangle, the only unitary spherical parameter is $\left(\frac{9}{2}, \frac{7}{2}\right)$, corresponding to the trivial representation.

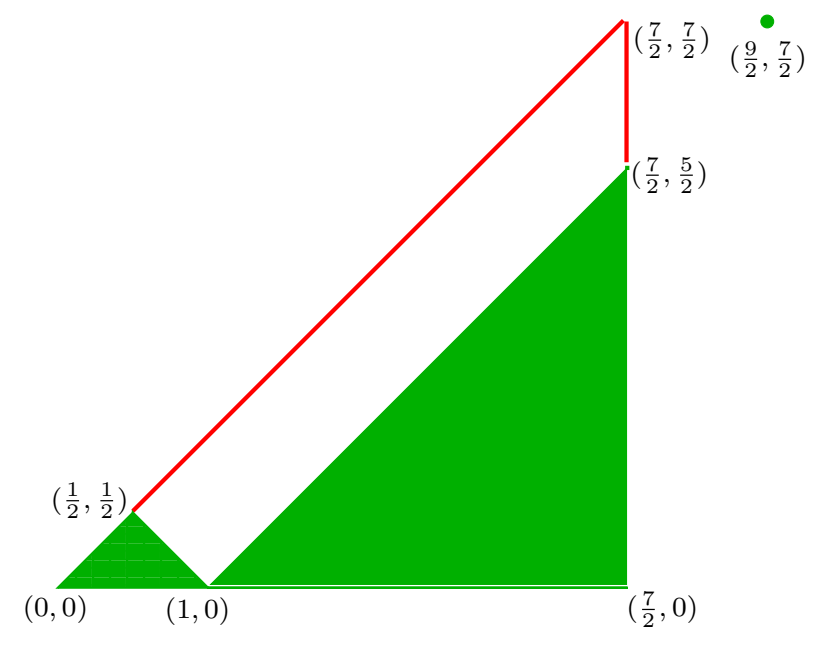

FiguRE 1. Spherical unitary parameters for $\mathbb{H}_{c}\left(B_{2}\right)$ with $c=7 / 2$ 


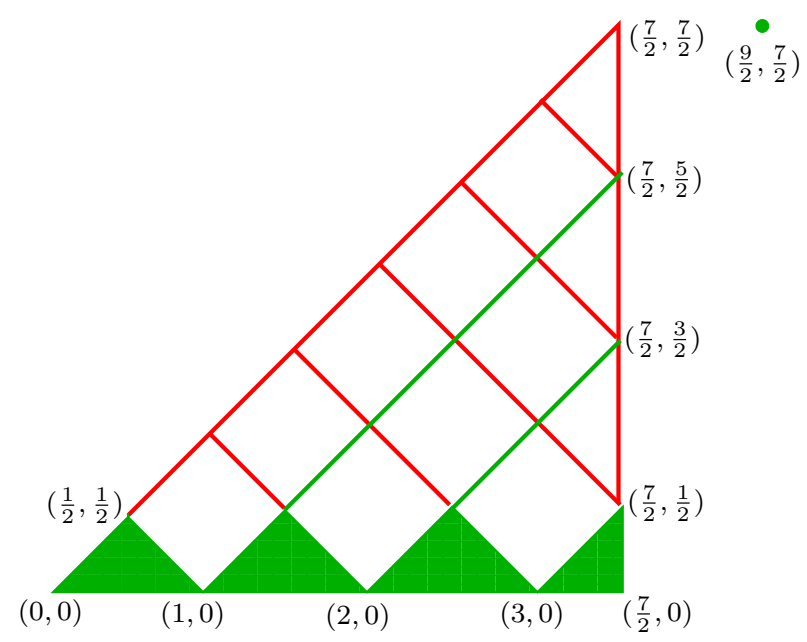

Figure 2. Spherical unitary parameters for $U(8,2)$

\section{REFERENCES}

[Ba1] D. Barbasch A reduction theorem for the unitary dual of $U(p, q)$, Noncommutative Harmonic Analysis, P. Delorme, M. Vergne editors, Progress in Mathematics, vol. 220, Birkhäuser, 2003, 21-60

[Ba2] The spherical unitary dual of split real and p-adic groups preprint, http://www.math.cornell.edu/ barbasch

[BC] D. Barbasch, D. Ciubotaru The spherical unitary dual of Hecke algebras of type E6, E7, and $E 8$ preprint, in preparation

[B] A. Borel Admissible representations of a semisimple group over a local field with fixed vectors under an Iwahori subgroup, Invent. Math., vol 35, 1976, 233-259

[BM1] D. Barbasch, A. Moy A unitarity criterion for p-adic groups Invent. Math., vol 98, 1989, 19-38

[BM2] Reduction to real infinitesimal character in affine Hecke algebras, Journal of the AMS, vol 6, Number 3, 1993, 611-635

[BM3]_Whittaker models with an Iwahori fixed vector, Representation theory and analysis on homogeneous spaces (New Brunswick, NJ, 1993), 101-105, Contemp. Math., 177, Amer. Math. Soc., Providence, RI, 1994.

[BV] D. Barbasch, D. Vogan Unipotent representations of complex semisimple groups Ann. of Math., 121, (1985), 41-110

[BK] C.J. Bushnell, P. Kutzko The admissible dual of $G L(N)$ via compact open subgroups Ann. of Math. Studies, Princeton Univ. Press, 1993

[BW] A. Borel, N. Wallach Continuous cohomology, discrete subgroups, and representations of reductive groups American Mathematical Society, Providence, RI, 1999

[C] D. Ciubotaru The Iwahori spherical unitary dual of the split group of type F4, Represent. Theory, vol 9, 2005, 94-137

[E] S. Evens Langlands classification for graded Hecke algebras, Proc. Amer. Math. Soc. 124 (1996), no. 4, 1285-1290

[HM] R. Howe, A. Moy Minimal K-types for Gl(n) over a p-adic field Orbites unipotentes et reprsentations, II, Astérisque No. 171-172, 1989, 257-273 
[KL] D. Kazhdan, G. Lusztig, Proof of the Deligne-Langlands conjecture for Hecke algebras, Inv Math., vol 87, $1987,153-215$

[K] J. Kim Hecke algebras of classical groups over p-adic fields and supercuspidal representations American Journal of Math., 121, no. 5, October 1999, 967-1029

[KS] A.W. Knapp, B. Speh, Theorems about unitary representations applicable for $S U(N, 2)$, Non-Commutative Harmonic Analysis, Springer Lecture Notes, vol. 1020. 1982

[KZ] A.W. Knapp, G. Zuckermann Classification theorems for representations of semisimple Lie groups, Non-commutative harmonic analysis (Actes Colloq., Marseille-Luminy, 1976), p. 138-159. Lecture Notes in Math., Vol. 587, Springer, Berlin, 1977.

[KR] C. Kriloff, A. Ram, Representations of graded Hecke algebras Represent. Theory no. 6, (2002), 31-69

[L1] G. Lusztig Characters of reductive groups over a finite field, Annals of Math. Studies, Princeton University Press, vol 107

[L2] _ Affine Hecke algebras and their graded version, Jour. AMS, vol 2, 1989, 599-635

[L3] _ Intersection cohomology complexes on a reductive group, Invent. Math., vol 75, 1984, 205-272

[L4] _ Cuspidal local systems and graded algebras I, Publ. Math de l'IHES, vol 67, 1988, $145-202$

[L5] _ Cuspidal local systems and graded algebras II, CMS Conf. Proc., 16, Representations of groups (Banff, AB, 1994), Amer. Math. Soc., Providence, RI, 1995, 217-275

[L6] Cuspidal local systems and graded algebras III, Represent. Theory no. 6, (2002), $202-242$

[L7] _ Classification of unipotent representations of simple p-adic groups Int. Math. Res. Notices 11, (1995), 517-589

[O] E. Opdam On the spectral decomposition of affine Hecke algebras Jour. of Inst. Math. Jussieu 3 no. 4, (2004), 531-648

[V1] D. Vogan, Unitarizability of certain series of representations, Ann. of Math., 120, 1984, 141-187

[V2] Gelfand-Kirillov dimension for Harish-Chandra modules, Invent. Math. 48, 1978, no. 1 , pp. $75-98$

[Y] J.K. Yu Construction of tame cuspidal representations Jour. Amer. Math. Soc., 14 no. 2 , 2001, 579-622

D. Barbasch

Dept. of Mathematics Cornell University Ithaca, NY 14850

E-mail: barbasch@math.cornell.edu

D. Ciubotaru

Dept. of Mathematics, MIT, Cambridge MA 02139

E-mail: ciubo@math.mit.edu 